\title{
A prey-predator fishery model with endogenous switching of harvesting strategy
}

\author{
Gian-Italo Bischi \\ Departments of Economics, Society, Politics \\ University of Urbino (Italy) \\ email: gian.bischi@uniurb.it \\ Fabio Lamantia \\ Department of Economics, Statistics and Finance \\ University of Calabria (Italy) \\ email: fabio.lamantia@unical.it \\ Davide Radi \\ "Lorenzo Mascheroni" Department of Mathematics, Statistics, Computing and Applications \\ University of Bergamo (Italy) \\ email: davide.radi@unibg.it
}

\begin{abstract}
We propose a dynamic model to describe a fishery where both preys and predators are harvested by a population of fishermen who are allowed to catch only one of the two species at a time. According to the strategy currently employed by each agent, i.e. the harvested variety, at each time period the population of fishermen is partitioned into two groups, and an evolutionary mechanism regulates how agents dynamically switch from one strategy to the other in order to improve their profits. Among the various dynamic models proposed, the most realistic is a hybrid system formed by two ordinary differential equations, describing the dynamics of the interacting species under fishing pressure, and an impulsive variable that evolves in a discrete time scale, in order to describe the changes of the fraction of fishermen that harvest a given stock. The aim of the paper is to analyze the economic consequences of this kind of self-regulating fishery, as well as its biological sustainability, in comparison with other regulatory policies. Our analytic and numerical results give evidence that in some cases this kind of myopic, evolutionary self-regulation might ensure a satisfactory trade-off between profit maximization and resource conservation.
\end{abstract}

Keywords: Fisheries management; Heterogeneous agents; Interacting populations; Evolutionary game theory; Hybrid dynamical systems.

\section{Introduction}

The exploitation of unregulated open access fisheries is characterized by a typical prisoner dilemma, often referred to as the 'tragedy of the commons' after [1]. As a consequence, individuals maximize short-term profits instead of pursuing long-term objectives with overexploitation of the resource and economic inefficiency, i.e. lower levels of resource and profits in the long run. 
Indeed, the sustainability of fisheries exploitation is constrained by the natural stocks growth as well as equilibrium patterns regulated by ecological interactions among species. Adding harvesting activity to such a complex (typically non linear) dynamical system opens scenarios which are not easy to be understood and managed. Moreover, the overexploitation of some fish stocks may have consequences for the whole ecosystem which are difficult to be forecasted, and may eventually lead to depletion of some species, and thus decreasing yields, up to the danger of unexpected extinction of resources. For these reasons, central institutions usually enforce forms of regulation either by imposing harvesting restrictions, such as constant efforts, individual fishing quotas, taxations, or by limiting the kinds of fish to be caught or the regions where exploitation is allowed (see e.g. [2], [3], [4], [5], [6]). Due to the peculiar issue, different sources of strategic interdependence among exploiters are present, as pointed out in [4], [5], [6], [7]. First, biological externalities must be taken into account, as overexploitation of the resource by some agents may have severe repercussions on the capacity of regeneration of the resource, thus giving a negative externality for the whole community of exploiters. Second, market externalities may exist, due to price reduction as a consequence of increasing harvesting, and finally cost externalities, due to the increase of harvesting costs when fish stock is depleted. On the basis of these self-regulating economic externalities, some experiments on endogenous regulatory policies have been recently performed, in which central authorities only establish some general rules and then fishermen are allowed to decide their fishing strategies according to short-period profit maximization arguments. For example, a recent law proposed in Italy to regulate the harvesting of two non interacting shellfishes (Venerupis aurea and Callista chione) in the Adriatic Sea, imposes that during a given time period (three years) each agent can harvest only one species, possibly revising the choice in predefined successive periods. In other words, instead of imposing a difficult-to-control policy (e.g. imposed effort, total allowable catch, etc.), the central authority just establishes that each vessel can harvest just a single kind of fish and has to stick to this choice for a given time interval. A first analysis of this model with two independent species has been carried out in $[8]$.

Along these ideas, in this paper we propose, as an exercise, a dynamic model to describe a situation where exploiters can harvest two different species which interact through a prey-predator relationship. According to the employed strategy, i.e. the target species, at each time the population of fishermen is partitioned into two groups. We first study the dynamics of the system in which these two groups do not change over time. Then, we introduce an evolutionary mechanism (replicator dynamics) based on the observed profits, which regulates how agents dynamically switch from one strategy to another. First we discuss the case in which this switching can take place continuously. Then we address the case of a discrete-time switching of the harvesting strategy (due to regulatory or logistic constraints).

Although discrete-time replicator models are know to generate more complicated behaviors in comparison with their continuous-time counterparts (see for instance [9]), we do not discretize the preypredator model because it is typically expressed in continuous time in biological modeling. Instead we embed the discrete replicator (decision driven) in a standard continuous-time model. In this case the model becomes a hybrid dynamical systems, i.e. a dynamical systems evolving in continuous time with some variables allowed to change at discrete times; moreover, these impulsive changes take place according to an endogenous switching mechanism. Hybrid dynamical systems are widely employed to 
describe engineering, biological and medical systems (see e.g. [10], [11], [12], [13]) and can also be of great interest in economic science.

The aim of the paper is to analyze, by analytical and numerical methods, the economic consequences of this kind of self-regulating fishery, as well as to shed some light on the sustainability of this form of exploitation in comparison to other policies. In the evolutionary game literature, it is well documented that common pool resource games can lead to overexploitation (or even extinction) when the Nash (myopic) strategy is played over time (see [14], [7]). However, here we show that the system could self-regulate even with the lack of cooperative behavior in the population of harvesters as a consequence of multi-species targets and economic and biological externalities. Indeed, our analysis gives evidence of possible advantages of profit-driven self regulated harvesting strategy choices over other practices, both from the point of view of biomass levels (i.e. biological sustainability) and profits (economic sustainability). Even far from the real system we aim at describing, the cases of nonevolutionary dynamics and evolutionary switching in continuous time provide useful suggestions about the directions of investigation for the more realistic hybrid system, as well as some intuitive interpretations of the properties observed through numerical simulation. Moreover, the simulation results suggest that this kind of myopic evolutionary regulation could in some cases ensure a virtuous trade-off between profit maximization and resource conservation.

The plan of the paper is as follows. In section 2 the prey-predator model is defined and the three harvesting functions employed in the paper are described: 1) imposed constant effort; 2) unrestricted harvesting; and 3) restricted harvesting; in the latter, the regulator only imposes that each agent is allowed to harvest one species at a time whereupon agents are free to decide their catch. In section 3 we study the dynamics of the prey-predator model with the various harvesting functions previously obtained, whereas in section 4 we analyze the evolutionary models both with continuous and discrete switching times. Numerical simulations of the dynamic equations described in sections 3 and 4 are compared in section 5. Section 6 concludes also providing suggestions for further work on the subject.

\section{The bioeconomic model}

Let us consider a marine ecosystem with two interacting fish species indexed by 1 and 2 with biomass (or density) measures $X_{1}$ and $X_{2}$ respectively, both subject to commercial harvesting. As customary, we assume that their time evolution is described by a two-dimensional continuous dynamical system of the form

$$
\begin{aligned}
& \dot{X}_{1}=X_{1} G_{1}\left(X_{1}, X_{2}\right)-H_{1}\left(X_{1}, X_{2}\right) \\
& \dot{X}_{2}=X_{2} G_{2}\left(X_{1}, X_{2}\right)-H_{2}\left(X_{1}, X_{2}\right)
\end{aligned}
$$

where $\dot{X}_{i}, i=1,2$, denote the time derivatives of biomass, $G_{i}$ specify the natural growth functions and $H_{i}$ represent the instantaneous harvesting of the two species.

Concerning the growth functions $G_{i}$, they may include different kinds of interspecific and intraspecific interactions (see e.g. [15], [16], [2], [17]). In the following, we focus on the well known Rosenzweig-MacArthur prey-predator model (see e.g. [18], [19], [20], [21], [22], [23]) characterized by 
saturation of predation uptake, described by a Holling type II functional response, given by

$$
G_{1}\left(X_{1}, X_{2}\right)=\rho\left(1-\frac{X_{1}}{K}\right)-\frac{\beta X_{2}}{\alpha+X_{1}} ; \quad G_{2}\left(X_{1}, X_{2}\right)=\frac{\eta \beta X_{1}}{\alpha+X_{1}}-d
$$

where $\rho$ is the intrinsic growth rate, $K$ is the environmental carrying capacity of prey population, $\beta$ is the maximum uptake rate for predator, $\eta$ denotes the ratio of biomass conversion (satisfying the obvious restriction $0<\eta<1$ ), $d$ is the natural death rate of predator and $\alpha$ represents the halfsaturation constant. All these parameters are assumed to be real and positive. Throughout the paper, we shall consider growth equations (2) such that the asymptotic behavior of the unexploited model (1) gives rise to coexistence of the two species, i.e. positive biomass values (stationary or oscillatory), letting available positive stocks of both species for sustainable intake.

In order to model the fishery, i.e. the harvesting functions $H_{i}$ in (1), let us assume that it is a common-pool resource and $N$ fishermen are allowed to land the stocks $X_{1}$ and $X_{2}$, and a regulator can manage the fishery in order to mitigate the effect of overexploitation. Besides the benchmark cases of constant effort imposed by the regulator and unrestricted harvesting of the stocks, the main case we develop in the paper involves a regulator which establishes 'weak' constraints on the fishery; namely, each fisherman must commit himself to harvesting only one kind of fish for a given period of time. Accordingly, the population of $N$ fishermen is partitioned into two groups of exploiters. We denote by $r \in[0,1]$ the fraction of agents harvesting species 1 , hence agents in the complementary fraction $(1-r)$ only catch species 2 . Let $h_{i}$ and $\pi_{i}$ be, respectively, the instantaneous biomass intake of species $i$ and the corresponding instantaneous profit of a representative agent in group $i, i=1,2$.

As for this fraction $r$, in the following we shall consider both the cases of constant exogenously imposed $r$ and endogenously updated $r=r(t)$. In the former case, the fraction of fishermen allowed to take a given species is imposed by the authority, according to some economic or social optimum criteria, whereas in the latter case this fraction is decided by the fishermen themselves, who are free to change the group they belong to over time. The cases of endogenously updated strategies with continuous and discrete time switchings are then developed in Section 4.

\subsection{Harvesting functions}

Here we examine the different harvesting functions that will be considered in the paper in order to model different exploitation behaviors regarding the two species.

\subsubsection{Imposed constant effort}

This is the simplest fisheries policy, where fishermen are allowed to harvest both stocks but a constant fishing effort $E$ is imposed by a central authority, so that the harvesting functions assume the form (see e.g. [2])

$$
H_{i}=q_{i} E X_{i}
$$

where $q_{i}$ is a technological coefficient and $E$ depends on the total number of vessels (each vessel is assumed to harvest both species with the same effort). Notice that, in principle, we should assume that the authority fixes a different effort level for each species. However, since in this model the catch 
is directly proportional to biomass, we assume that this difference is included in the specific intake factor $q_{i} E$.

\subsubsection{Unrestricted harvesting}

Here we assume that the $N$ fishermen, acting as oligopolists, are all free to harvest both kinds of fish (preys and predators), whose current total stocks are, respectively, $X_{1}$ and $X_{2}$. Since no constraints are imposed, we assume that agents are all homogeneous. We recall that $h_{1}$ and $h_{2}$ denote the quantities of the two species harvested by each representative agent.

Following [24] and [25], we assume a linear demand system defining the current selling prices of the two species as

$$
p_{1}=a_{1}-b_{1} N\left(h_{1}+\sigma h_{2}\right) ; \quad p_{2}=a_{2}-b_{2} N\left(\sigma h_{1}+h_{2}\right)
$$

where $a_{i}$ and $b_{i}$ represent, respectively, the maximum price consumers are willing to pay and the slope of the demand for species $i ; \sigma \in[0,1]$ is the symmetric degree of substitutability between the two fish varieties: if $\sigma=0$ the two varieties are independent in demand, on the other hand for $\sigma=1$ they are perfect substitutes (we disregard the case $\sigma<0$ modeling varieties that are demand complementary). Many authors (see again [24] and [25]) assume $b_{1}=b_{2}=b$.

Concerning cost functions, as standard in models of fisheries we assume quadratic harvesting costs for both species ${ }^{1}$, i.e.

$$
C_{i}\left(X_{i}, h_{i}\right)= \begin{cases}\gamma_{i} \frac{h_{i}^{2}}{X_{i}} & \text { if } X_{i}>0 \\ 0 & \text { if } X_{i}=0\end{cases}
$$

where $\gamma_{i}$ is a technological parameter for catching species $i$. This cost function can be derived from a Cobb-Douglas type "production function" with fishing effort (labor) and fish biomass (capital) as production inputs (see e.g. [26], [2]). It captures the fact that it is easier and less expensive to catch fish if the fish population is large, so that it includes resource stock externalities.

The profit of the representative fisherman is

$$
\pi=\left[a_{1}-b_{1} N\left(h_{1}+\sigma h_{2}\right)\right] h_{1}+\left[a_{2}-b_{2} N\left(\sigma h_{1}+h_{2}\right)\right] h_{2}-\gamma_{1} \frac{h_{1}^{2}}{X_{1}}-\gamma_{2} \frac{h_{2}^{2}}{X_{2}}
$$

As standard in game-theoretic models, each agent makes his/her own choice by considering that also other agents are profit maximizers. The harvesting quantities $h_{i}^{*}$ which maximize the instantaneous profit are given by

$$
h_{i}^{*}=\frac{a_{j}\left(b_{j}+N b_{i}\right) X_{i} X_{j} \sigma-a_{i} X_{i}\left(b_{j}(1+N) X_{j}+2 \gamma_{j}\right)}{\left(b_{i}+N b_{j}\right)\left(b_{j}+N b_{i}\right) X_{i} X_{j} \sigma^{2}-\left(b_{i}(1+N) X_{i}+2 \gamma_{i}\right)\left(b_{j}(1+N) X_{j}+2 \gamma_{j}\right)} ; i, j=1,2 ; i \neq j
$$

In the trivial case of $X_{i}=0$, we set $h_{i}^{*}=0$ throughout the paper.

By inserting (7) into (6) the optimal individual profit becomes

$$
\pi^{*}=\left(b_{1}+\frac{\gamma_{1}}{X_{1}}\right)\left(h_{1}^{*}\right)^{2}+\left(b_{2}+\frac{\gamma_{2}}{X_{2}}\right)\left(h_{2}^{*}\right)^{2}+h_{1}^{*} h_{2}^{*} \sigma\left(b_{1}+b_{2}\right)
$$

\footnotetext{
${ }^{1}$ The adopted notation emphasizes that costs (as well as harvesting and profits) are equal to zero whenever $X_{i}=0$.
} 
An assumption to get a more tractable algebra consists in letting $b=0$, i.e. perfectly elastic demands with fixed prices $p_{i}=a_{i}$. The assumption of fixed prices is often justified by the fact that there are many substitutes for each species and fish is considered a staple food for most consumers. With fixed prices, individual optimal harvesting and profits read:

$$
h_{i}^{*}=\frac{a_{i} X_{i}}{2 \gamma_{i}}, \quad \pi^{*}=\gamma_{1} \frac{h_{1}^{* 2}}{X_{1}}+\gamma_{2} \frac{h_{2}^{* 2}}{X_{1}}=\frac{a_{1}^{2} X_{1}}{4 \gamma_{1}}+\frac{a_{2}^{2} X_{2}}{4 \gamma_{2}}
$$

Therefore with unrestricted harvesting, total industry catch is $H_{i}=N h_{i}^{*}$.

\subsubsection{Restricted harvesting}

Here we consider $N$ fishermen divided into two groups, say group 1 and 2 , such that a fisherman belonging to group $i$ can only harvest fish of species $i$. Let $h_{i}$ be the actual quantity of species $i$ harvested by the representative agent of group $i$. We recall that $r$ denotes the fraction of agents harvesting species 1 . The linear demand system becomes

$$
\begin{aligned}
& p_{1}=a_{1}-b_{1} N\left(r h_{1}+\sigma(1-r) h_{2}\right) \\
& p_{2}=a_{2}-b_{2} N\left(\sigma r h_{1}+(1-r) h_{2}\right)
\end{aligned}
$$

where the constant $a_{i}$ and $\sigma$ have been defined in Subsection 2.1.2. The profit function for the representative agent harvesting species $i$ is

$$
\pi_{i}=p_{i} h_{i}-C_{i}\left(X_{i}, h_{i}\right)=\left[a_{i}-b_{i} N\left(r_{i} h_{i}+\sigma r_{j} h_{j}\right)\right] h_{i}-\gamma_{i} \frac{h_{i}^{2}}{X_{i}}, i, j=1,2, i \neq j
$$

where $r_{1}=r, r_{2}=1-r$ and with cost functions (5). The 'optimal' instantaneous harvesting level $h_{i}^{*}$ for species $i$ is

$$
h_{i}^{*}=\frac{a_{i} X_{i}\left(b_{j} X_{j}\left[1+N r_{j}\right]+2 \gamma_{j}\right)-a_{j} b_{i} N r_{j} X_{i} X_{j} \sigma}{\left(b_{i} X_{i}\left(1+N r_{i}\right)+2 \gamma_{i}\right)\left(b_{j} X_{j}\left(1+N r_{j}\right)+2 \gamma_{j}\right)-b_{i} b_{j} N^{2} r_{i} r_{j} X_{i} X_{j} \sigma^{2}}, i, j=1,2, i \neq j
$$

By inserting (11) into (10), we get optimal individual profits

$$
\pi_{i}^{*}=\left(b_{i}+\frac{\gamma_{i}}{X_{i}}\right)\left(h_{i}^{*}\right)^{2}
$$

Also in this case, the assumption of perfectly elastic demands for both stocks $(b=0)$ allows us to get a simpler expression of the individual optimal harvesting and individual profits, given by

$$
h_{i}^{*}=\frac{a_{i} X_{i}}{2 \gamma_{i}}, \quad \pi_{i}^{*}=\frac{a_{i}^{2} X_{i}}{4 \gamma_{i}}
$$

and total industry profit

$$
\pi^{*}=N\left[r \pi_{1}^{*}+(1-r) \pi_{2}^{*}\right]
$$

respectively. Notice that, as expected, the total instantaneous profits are greater in the case of unrestricted harvesting (compare (8) and (14)). In order to capture the effects of the different harvesting strategies on the ecosystem as well as the time evolution of profits, we now consider the dynamic models of the fishery system (1) with the different harvesting functions obtained in this section. 


\section{The non-evolutionary dynamic models}

We now consider the general dynamic model (1) with the three different harvesting functions proposed in the previous section, in order to compare the different time evolutions of the ecological system and profits. In particular, in this section, the case of harvesting restricted to one species at a time is analyzed assuming that the proportion of agents exploiting the two stocks is ex-ante decided by an authority and held fixed, i.e. we analyze a non-evolutionary version of the model. The evolutionary counterpart is then considered in Section 4.

\subsection{The dynamic model with undifferentiated constant effort harvesting}

We first analyze the model (1) obtained under the assumption of imposed constant effort $E \geq 0$, i.e. with harvesting functions (3). The time evolution of the fish biomasses is thus modelled by the following system of differential equations

$$
\begin{aligned}
& \dot{X}_{1}=\rho X_{1}\left(1-\frac{X_{1}}{K}\right)-\frac{\beta X_{1} X_{2}}{\alpha+X_{1}}-q_{1} E X_{1} \\
& \dot{X}_{2}=X_{2}\left(\frac{\eta \beta X_{1}}{\alpha+X_{1}}-d\right)-q_{2} E X_{2}
\end{aligned}
$$

The model is practically the same as the classical Rosenzweig-MacArthur prey-predator model (see e.g. [18], [19], [27], [28], [29]) with linear extra mortality terms both in prey and predator equations. So, simply translating the results given in the quoted references we obtain the following dynamic scenario.

Proposition 1. The dynamical system (15) has three non-negative equilibria: $(0,0),\left(\frac{K\left(\rho-q_{1} E\right)}{\rho}, 0\right)$,

$$
\left(\bar{X}_{1}^{E},{\overline{X_{2}}}^{E}\right)=\left(\frac{\alpha\left(d+q_{2} E\right)}{\eta \beta-d-q_{2} E}, \frac{\alpha \eta \beta}{K \beta}\left(\frac{\left(\rho-q_{1} E\right) K\left(\eta \beta-d-q_{2} E\right)-\rho \alpha\left(d+q_{2} E\right)}{\left(\eta \beta-d-q_{2} E\right)^{2}}\right)\right)
$$

The equilibrium $\left(\frac{K\left(\rho-q_{1} E\right)}{\rho}, 0\right)$ is positive as long as $\rho>q_{1} E$ and is a saddle point if the coexistence equilibrium $\left({\overline{X_{1}}}^{E},{\overline{X_{2}}}^{E}\right)$ is in the positive orthant. The coexistence equilibrium $\left({\overline{X_{1}}}^{E},{\overline{X_{2}}}^{E}\right)$ belongs to the positive orthant iff

$$
\eta \beta>d+q_{2} E, \quad \text { and } \rho-q_{1} E>\frac{\rho \alpha\left(d+q_{2} E\right)}{K\left(\eta \beta-d-q_{2} E\right)}
$$

and it is stable for

$$
\rho \alpha\left(d+q_{2} E\right)<K\left(\rho-q_{1} E\right)\left(\eta \beta-d-q_{2} E\right)<\rho \alpha\left(\eta \beta+d+q_{2} E\right)
$$

Notice that the second condition in (16) implies that the coexisting equilibrium exists only if $\rho$ is sufficiently higher than $q_{1} E$. In analogy with the case of unexploited model (see e.g. [22]) the following bifurcation curves are defined in the parameters' space

$$
\begin{aligned}
& K=K_{T}=\frac{\rho \alpha\left(d+q_{2} E\right)}{\left(\rho-q_{1} E\right)\left(\eta \beta-d-q_{2} E\right)} \text { (transcritical bifurcation curve) } \\
& K=K_{H}=K_{T}+\frac{\rho \alpha \eta \beta}{\left(\rho-q_{1} E\right)\left(\eta \beta-d-q_{2} E\right)} \text { (Hopf bifurcation curve) }
\end{aligned}
$$



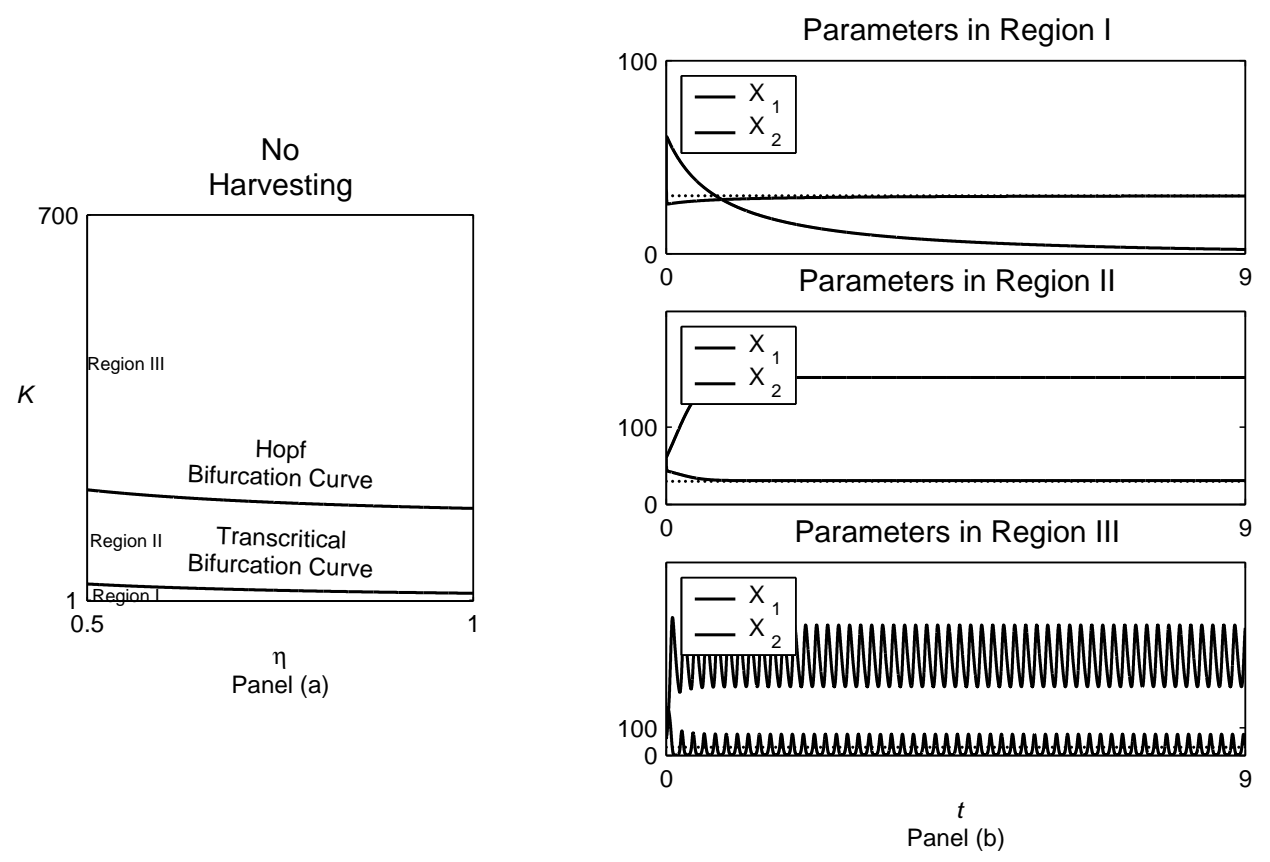

Figure 1: (a) Bifurcation curves in the parameters' space $(\eta, K)$ for the prey-predator model without harvesting $(E=0)$ and parameters' values: $\rho=250, \beta=100, \alpha=140, d=9$. The regions bounded by the bifurcation curves are denoted as region I (predator extinction region), region II (stable coexistence equilibrium) and region III (oscillatory coexistence along a limit cycle). (b) Time evolution of three typical trajectories, one for each region. The dotted lines in panel (b) represent the carrying capacity of the prey.

In Fig. 1, the bifurcation curves in the reference case of no harvesting (i.e. $E=0$ ) are represented, as well as the regions bounded by them, denoted as region I (predator extinction region), region II (stable coexistence equilibrium) and region III (oscillatory coexistence along a limit cycle). Three typical time evolutions, one for each region, are also represented in Fig. 1b. Instead, Fig. 2a exhibits the same bifurcation curves obtained with $E>0$.

\subsection{Dynamic fishery with unrestricted harvesting}

Here we consider the model (1) with harvesting functions (7). Under the assumption $b=0$, i.e. fixed prices, the harvesting functions are given in (8) and the model becomes

$$
\begin{aligned}
& \dot{X}_{1}=\rho X_{1}\left(1-\frac{X_{1}}{K}\right)-\frac{\beta X_{1} X_{2}}{\alpha+X_{1}}-N \frac{a_{1} X_{1}}{2 \gamma_{1}} \\
& \dot{X}_{2}=X_{2}\left(\frac{\eta \beta X_{1}}{\alpha+X_{1}}-d\right)-N \frac{a_{2} X_{2}}{2 \gamma_{2}}
\end{aligned}
$$

for which the following results can be proved (see Appendix A).

Proposition 2. The dynamical system (19) has three non-negative equilibrium points, given by 

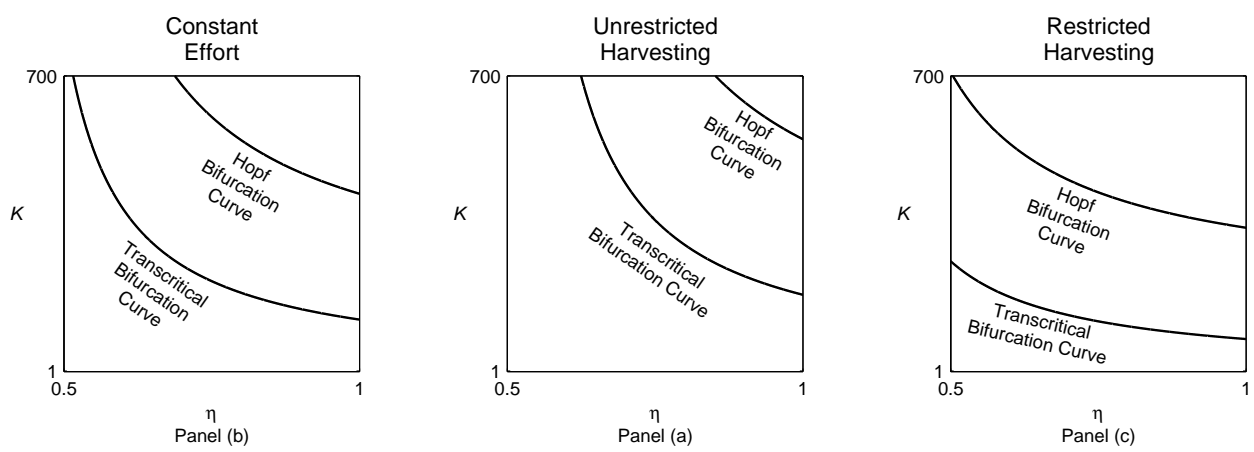

Figure 2: (a) Bifurcation curves with constant fishing effort $E_{1}=40 \frac{a_{1}}{2 \gamma_{1}}, E_{2}=40 \frac{a_{2}}{2 \gamma_{2}}, q_{1}=q_{2}=1$. (b) Bifurcation curves with unrestricted harvesting, biological parameters as in Fig. 1 and economic parameters: $\gamma_{1}=4, \gamma_{2}=6.2, N=50, a_{1}=a_{2}=10$. (c) Bifurcation curves with exploiters split equally in two groups, i.e. $r=0.5$ (fixed).

$S_{0}=(0,0), S_{1}=\left(\frac{K\left(2 \rho \gamma_{1}-N a_{1}\right)}{2 \rho \gamma_{1}}, 0\right)$, provided that $2 \rho \gamma_{1}>N a_{1}$, and $S_{2}=\left(X_{1}^{*}, X_{2}^{*}\right)$ with

$$
X_{1}^{*}=\alpha \frac{2 \gamma_{2} d+N a_{2}}{2 \gamma_{2} \eta \beta-2 \gamma_{2} d-N a_{2}} \text { and } X_{2}^{*}=\frac{X_{1}^{*}+\alpha}{K \beta}\left(\rho\left(K-X_{1}^{*}\right)-K N \frac{a_{1}}{2 \gamma_{1}}\right)
$$

that has non-negative components provided that

$$
\eta \beta>d+N \frac{a_{2}}{2 \gamma_{2}} \quad \text { and } 2 \rho \gamma_{1}-N a_{1}>\frac{2 \rho \gamma_{1} \alpha\left(d+N \frac{a_{2}}{2 \gamma_{2}}\right)}{K\left(\eta \beta-d-N \frac{a_{2}}{2 \gamma_{2}}\right)}
$$

At $\frac{\eta \beta K\left(2 \rho \gamma_{1}-N a_{1}\right)}{K\left(2 \rho \gamma_{1}-N a_{1}\right)+2 \rho \gamma_{1} \alpha}-d-N \frac{a_{2}}{2 \gamma_{2}}=0$, i.e. at

$$
K=K_{T}^{f}=\frac{2 \rho \gamma_{1} \alpha\left(d+\frac{N a_{2}}{2 \gamma_{2}}\right)}{\left(2 \rho \gamma_{1}-N a_{1}\right)\left(\eta \beta-d-\frac{N a_{2}}{2 \gamma_{2}}\right)}
$$

a transcritical bifurcation occurs, at which the equilibrium $S_{2}$ enters the positive orthant and $S_{1}$ becomes a saddle point, whereas at $K\left(2 \rho \gamma_{1}-N a_{1}\right)\left(\eta \beta-d-N \frac{a_{2}}{2 \gamma_{2}}\right)-2 \rho \gamma_{1} \alpha\left(\eta \beta+d+N \frac{a_{2}}{2 \gamma_{2}}\right)=0$, i.e. at

$$
K=K_{H}^{f}=K_{T}^{f}+\frac{2 \rho \gamma_{1} \alpha \eta \beta}{\left(2 \rho \gamma_{1}-N a_{1}\right)\left(\eta \beta-d-\frac{N a_{2}}{2 \gamma_{2}}\right)}
$$

the equilibrium $S_{2}$ loses stability through a supercritical Hopf Bifurcation.

\subsection{Dynamics with restricted harvesting}

Here we consider the model (1) with the harvesting functions of Subsection 2.1.3, where $r \in[0,1]$ is an exogenous parameter. Again, in order to obtain some analytical results, we study the model with fixed prices, i.e. with harvesting functions (13), thus having: 


$$
\begin{aligned}
& \dot{X}_{1}=\rho X_{1}\left(1-\frac{X_{1}}{K}\right)-\frac{\beta X_{1} X_{2}}{\alpha+X_{1}}-r N \frac{a_{1} X_{1}}{2 \gamma_{1}} \\
& \dot{X}_{2}=X_{2}\left(\frac{\eta \beta X_{1}}{\alpha+X_{1}}-d\right)-(1-r) N \frac{a_{2} X_{2}}{2 \gamma_{2}}
\end{aligned}
$$

The following characterization of equilibrium points holds (see Appendix A for a proof):

Proposition 3. The dynamical system (22) has three non-negative equilibrium points, given by $S_{0}=(0,0), S_{1}^{r}=\left(\frac{K\left(2 \rho \gamma_{1}-r N a_{1}\right)}{2 \rho \gamma_{1}}, 0\right)$ and $S_{2}^{r}=\left(X_{1}^{r}, X_{2}^{r}\right)$, with $X_{1}^{r}=\frac{\alpha\left(d+(1-r) N \frac{a_{2}}{2 \gamma_{2}}\right)}{\eta \beta-d-(1-r) N \frac{a_{2}}{2 \gamma_{2}}}, X_{2}^{r}=$ $\frac{\left(\alpha+X_{1}^{r}\right)}{\beta}\left[\rho-\frac{\rho X_{1}^{r}}{K}-r N \frac{a_{1}}{2 \gamma_{1}}\right]$.

The Equilibrium $S_{1}^{r}$ is positive if $2 \rho \gamma_{1}>r N a_{1}$, and $S_{2}^{r}$ is positive provided that $\eta \beta>d+$ $(1-r) N \frac{a_{2}}{2 \gamma_{2}}$ and $X_{1}^{r}<\frac{K\left(2 \rho \gamma_{1}-r N a_{1}\right)}{2 \rho \gamma_{1}}$.

$S_{2}^{r}$ becomes stable through a transcritical bifurcation at which $S_{1}^{r}$ and $S_{2}^{r}$ exchange stability, and loses stability through a supercritical Hopf bifurcation; the analytical expressions for bifurcations curves are given by

$$
\begin{aligned}
& K=K_{T}^{r}=\frac{2 \rho \gamma_{1} \alpha\left(d+(1-r) \frac{a_{2} N}{2 \gamma_{2}}\right)}{\left(2 \rho \gamma_{1}-N a_{1}\right)\left(\eta \beta-d-(1-r) \frac{a_{2} N}{2 \gamma_{2}}\right)} \text { (Transcritical bifurcation curve) } \\
& K=K_{H}^{r}=K_{T}^{r}+\frac{2 \rho \gamma_{1} \alpha \eta \beta}{\left(2 \rho \gamma_{1}-N a_{1}\right)\left(\eta \beta-d-(1-r) \frac{a_{2} N}{2 \gamma_{2}}\right)} \text { (Hopf bifurcation curve) }
\end{aligned}
$$

A graphical representation of the local bifurcation curves obtained is reported in Fig. 2: the central panel shows the bifurcation curves and the stability regions for the case of unrestricted oligopolistic competition (Proposition 2), whereas Fig. 2c depicts the same curves and regions for the model with intake restricted to one species (Proposition 3). Visual inspection reveals that the transcritical bifurcation curve is shifted down in the latter case, so that the region of coexistence (region II plus region III) is wider in the latter case.

\section{Evolutionary dynamics}

In this section and in the next one, we analyze the case where fishermen are allowed to choose which species they prefer to harvest on the basis of the observed profits, i.e. they can switch from a fishing strategy to the one expected to be more profitable. Thus, $r$ is no longer a fixed parameter but it becomes an endogenous dynamic variable.

We start our study with the case of continuous time replicator dynamics (see [30], [31], [14]), modelled through the following nonlinear three-dimensional system of ODE

$$
\begin{aligned}
\dot{X}_{1} & =X_{1} G_{1}\left(X_{1}, X_{2}\right)-N r(t) h_{1}^{*}\left(X_{1}, X_{2}\right) \\
\dot{X}_{2} & =X_{2} G_{2}\left(X_{1}, X_{2}\right)-N(1-r(t)) h_{2}^{*}\left(X_{1}, X_{2}\right) \\
\dot{r} & =r\left[\pi_{1}-\left(r \pi_{1}+(1-r) \pi_{2}\right)\right]=r(1-r)\left[\pi_{1}-\pi_{2}\right]
\end{aligned}
$$


where $h_{i}^{*}, i=1,2$ are the instantaneous intakes of species $i$ given in (11), which maximize expected the instantaneous profit $\pi_{i}$, so that the harvesting terms in (1) become $H_{1}=N r h_{1}^{*}$ and $H_{2}=N(1-r) h_{2}^{*}$ respectively.

However, in real systems the authority imposes that fishermen have to commit themselves to the decided strategy for a given period of time $s>0$ (switching time). Thus we consider a more realistic description of this type of endogenous evolutionary adjustment mechanism through an hybrid dynamic model with discrete-time (or impulsive) switching. Fishermen decide these updates on the basis of observed profits, thus giving rise to endogenous evolutionary dynamics, according to the replicator equation in discrete time (see [30], [31], [7]). This leads to a dynamic model with continuous-time growth and harvesting of the fish species and discrete (or pulse) strategy switching. Thus the last equation in (25) is replaced by

$$
r(t)=\left\{\begin{array}{cc}
r(t-s) \frac{\bar{\pi}_{1}}{r(t-s) \bar{\pi}_{1}+[1-r(t-s)] \bar{\pi}_{2}} & \text { if } \frac{t}{s}=\left\lfloor\frac{t}{s}\right\rfloor \\
r\left(\left\lfloor\frac{t}{s}\right\rfloor s\right) & \text { otherwise }
\end{array} \text { where } \bar{\pi}_{i}=\frac{\int_{t-s}^{t} \pi_{i}(\tau) d \tau}{s}\right.
$$

where $\lfloor x\rfloor$ is the largest integer not greater than $x$ (i.e. the floor of $x$ ) and $\bar{\pi}_{i}$ represents the average profit of fishermen that harvested species $i$ in the previous period. Notice that in the limiting case $s \rightarrow 0$, equation (26) becomes the replicator equation with continuous-time switching (25).

\subsection{Profit driven replicator dynamics in continuous time}

In this case the dynamic model is given by a system of three ordinary differential equations: the usual two equations of biomass dynamics in (1) and the third one of the replicator dynamics in (25), which regulates the time evolution of the fraction of fishermen choosing to harvest species 1 , where $h_{i}^{*}$ and $\pi_{i}^{*}$ are given, respectively, by (11) and (12). Notice that the set $r \in[0,1]$ is a trapping region, that is, if the initial condition $r(0) \in[0,1]$, then $r(t) \in[0,1]$ for all values of $t \geq 0$. Moreover, $r=0$ and $r=1$ are trapping surfaces, that is, if $r(0)=0$ then $r(t)=0$ for all $t \geq 0$; the analogous property holds for $r=1$. From the replicator equation in (25), we have that the equilibria of the system must be located in the trapping regions $r=1, r=0$ or in the isoprofit surface $\pi_{1}^{*}=\pi_{2}^{*}$. However, due to the complicated algebraic expressions of $h_{i}^{*}$ and $\pi_{i}^{*}$, an analysis of the conditions for existence and stability of the equilibrium points is quite difficult in the general case. Therefore, we rely on numerical simulations in section 5 to explore the dynamics of this three-dimensional dynamical system.

In the remainder of this subsection, we consider the simpler case of constant prices, i.e. $b_{1}=b_{2}=0$. In this case, according to (13), the dynamic model is described by the following system of ODEs:

$$
\begin{aligned}
\dot{X}_{1} & =\rho X_{1}\left(1-\frac{X_{1}}{K}\right)-\frac{\beta X_{1} X_{2}}{\alpha+X_{1}}-r N \frac{a_{1} X_{1}}{2 \gamma_{1}} \\
\dot{X}_{2} & =X_{2}\left(\frac{\eta \beta X_{1}}{\alpha+X_{1}}-d\right)-(1-r) N \frac{a_{2} X_{2}}{2 \gamma_{2}} \\
\dot{r} & =r(1-r)\left(\frac{a_{1}^{2}}{4 \gamma_{1}} X_{1}-\frac{a_{2}^{2}}{4 \gamma_{2}} X_{2}\right)
\end{aligned}
$$

The analysis of the equilibria of the model are given below. 
Proposition 4. The system of ordinary differential equations (27) admits the following equilibria: $S_{0}^{e}=(0,0, r)$, with $r \in[0,1]$;

$S_{1}^{e}=(K, 0,0)$;

$S_{2}^{e}=\left(\frac{K\left(2 \rho \gamma_{1}-N a_{1}\right)}{2 \rho \gamma_{1}}, 0,1\right)$, with $2 \rho \gamma_{1}>N a_{1}$;

$S_{3}^{e}=\left(X_{1}^{e}, X_{2}^{e}, 0\right)$, where $X_{1}^{e}=\frac{\alpha\left(N a_{2}+2 d \gamma_{2}\right)}{2 \gamma_{2}(\eta \beta-d)-N a_{2}}$ and $X_{2}^{e}=\frac{\rho\left(\alpha+X_{1}^{e}\right)}{\beta}\left[1-\frac{X_{1}^{e}}{K}\right]$;

$S_{4}^{e}=\left(\widetilde{X}_{1}^{e}, \widetilde{X}_{2}^{e}, 1\right)$, where $\widetilde{X}_{1}^{e}=\frac{d \alpha}{\eta \beta-d}$ and $\widetilde{X}_{2}^{e}=\frac{\left(\alpha+\widetilde{X}_{1}^{e}\right)}{\beta}\left[\rho-\frac{\widetilde{X}_{1}^{e}}{K}-N \frac{a_{1}}{2 \gamma_{1}}\right]$;

$S_{5}^{e}=\left(\widehat{X}_{1}^{e}, \widehat{X}_{2}^{e}, \widehat{r}\right)$, where $\widehat{X}_{1}^{e}=\alpha \frac{2 d \gamma_{2}+(1-\widehat{r}) N a_{2}}{2 \eta \beta \gamma_{2}-2 d \gamma_{2}-(1-\widehat{r}) N a_{2}}$ and $\widehat{X}_{2}^{e}=\frac{\gamma_{2} a_{1}^{2}}{\gamma_{1} a_{2}^{2}} \widehat{X}_{1}^{e}$, where $\widehat{r}$ can assume at most two values inside $(0,1)$ given by the real solutions (if any) of a second degree algebraic equation.

The global extinction equilibria $S_{0}^{e}$ are stable if $2 \gamma_{1} \rho<r N a_{1}$; the equilibrium $S_{1}^{e}$, with predator's extinction and no prey harvesting, is always unstable; the equilibrium $S_{2}^{e}$, with predator's extinction and all fishermen harvesting preys, is unstable if $K\left(2 \rho \gamma_{1}-N a_{1}\right)(\eta \beta-d)>2 d \rho \gamma_{1} \alpha$; the equilibrium $S_{3}^{e}$, with coexisting preys and predators and no prey harvesting, is stable if $\pi_{1}^{*}<\pi_{2}^{*}, \beta \in\left(\frac{N a_{2}+2 d \gamma_{2}}{2 \gamma_{2} \eta},+\infty\right)$ and $K \in\left(0,-\frac{\alpha\left(N a_{2}+2 \gamma_{2}(d+\beta \eta)\right)}{N a_{2}+2 \gamma_{2}(d-\beta \eta)}\right)$; the equilibrium $S_{4}^{e}$, with coexisting preys and predators and all fishermen harvesting preys, is stable if $\pi_{1}^{*}>\pi_{2}^{*}$, and $K \in\left(0, \frac{\alpha(d+\beta \eta)}{\beta \eta-d}\right]$ or, when $K \in\left(\frac{\alpha(d+\beta \eta)}{\beta \eta-d},+\infty\right)$, for $\rho d \frac{(d(K+\alpha)-(\alpha-K) \beta \eta)}{K \beta \eta(d-\beta \eta)}<\frac{N a_{1}}{2 \gamma_{1}}$; the equilibria (if any) $S_{5}^{e}$ where both prey and predators are harvested, is unstable if $\frac{\alpha \beta \gamma_{2} a_{1}^{2} \widehat{X}_{1}^{e}}{\gamma_{1} a_{2}^{2}\left(\alpha+\widehat{X}_{1}^{e}\right)^{2}} \geq \frac{\rho}{K}$ whereas, if the reverse inequality holds, it is possible to find suitable parameter values such that $S_{5}^{e}$ is stable.

Proof and details are in Appendix B.

It is worth noticing that the most interesting equilibrium is $S_{5}^{e}$, as it is characterized by harvesting of both stocks (so that consumers can found both fish species in the market) with a given proportion defined by the isoprofit condition $X_{2}=\frac{\gamma_{2} a_{1}^{2}}{\gamma_{1} a_{2}^{2}} X_{1}$. The isoprofit condition has a clear economic meaning, and the parameters involved can be easily controlled by properly tuning cost and price parameters.

\subsection{Discrete time impulsive switching based on profit driven replicator dynamics}

We finally consider the model (26) characterized by stocks dynamics and harvesting activities in continuous time with strategy switches at discrete decision-driven times; the length $s$ of the time interval between decisions is the only form of regulatory policy in the model. In particular, we deal with the dynamical system (26) where $h_{i}^{*}$ and $\pi_{i}^{*}$ are given, respectively, in (11) and (12). Assuming again constant prices, i.e. $b=0$, the dynamical system reads

$$
\begin{aligned}
& \dot{X}_{1}=\rho X_{1}\left(1-\frac{X_{1}}{K}\right)-\frac{\beta X_{1} X_{2}}{\alpha+X_{1}}-r N \frac{a_{1} X_{1}}{2 \gamma_{1}} \\
& \dot{X}_{2}=X_{2}\left(\frac{\eta \beta X_{1}}{\alpha+X_{1}}-d\right)-(1-r) N \frac{a_{2} X_{2}}{2 \gamma_{2}} \\
& r(t)=\left\{\begin{array}{cr}
r(t-s)\left(\frac{a_{1}^{2} X_{1}}{4 \gamma_{1}}\right)\left(\frac{4 \gamma_{1}}{r(t-s) a_{1}^{2} X_{1}}+\frac{4 \gamma_{2}}{[1-r(t-s)] a_{2}^{2} X_{2}}\right) & \text { if } \frac{t}{s}=\left\lfloor\frac{t}{s}\right\rfloor \\
r\left(\left\lfloor\frac{t}{s}\right\rfloor s\right) & \text { otherwise }
\end{array}\right.
\end{aligned}
$$

Of course, any equilibrium point for the evolutionary model in continuous time (see Proposition 4) is also an equilibrium for the hybrid system (28), because the first and the second dynamic equations are identical, and the replicator dynamics in discrete time has the same equilibrium conditions being 
$r(t)=r(t-s)$ for $r=0, r=1$ or $\pi_{1}^{*}=\pi_{2}^{*}$. However, the converse is not necessarily true. In fact, in the hybrid model an equilibrium is characterized by the condition that the average profits of the two strategies over the interval $s$ are equal, but instantaneous profits could differ over time.

Some insights on the dynamics of model (28) and the comparison with the other benchmarks are given in next section. As we shall see, $r(t)$ becomes a piecewise-constant function, like an endogenously driven bang-bang parameter whose discontinuous jumps occur at discrete times and leads to sudden switch among different dynamic scenarios, which is a typical behavior of hybrid systems, see e.g. [32], [13], [33].

\section{$5 \quad$ Numerical Simulations}

In this section we propose the results of some numerical explorations of the different models described in the previous sections, in order to compare the different exploitation behaviors both from the biological and the economic point of view. All the numerical simulations shown in this section are essentially obtained by using a reference constellation of parameters, and only the two bifurcation parameters $K$ and $\eta$ are varied. However, the dynamic scenarios observed are representative of the behaviors we observed in many more cases.

A typical trajectory of the prey-predator model without harvesting is depicted in Fig. 3a, where the biological parameters are set as follows: $\rho=250, K=140, \alpha=140, \beta=100, \eta=0.6, d=9$. According to Proposition 1, an oscillatory convergence to the coexistence equilibrium $\left({\overline{X_{1}}}^{E},{\overline{X_{2}}}^{E}\right)$ is obtained. Now let us suppose that the Fishing Authority decides to give 50 licences for fishing both preys and predators according to the unrestricted oligopolistic competition described in section 2.1.2, with economic parameters $\gamma_{1}=4, \gamma_{2}=6.2, \sigma=0.5, a_{1}=a_{2}=10, b_{1}=b_{2}=0$. In Fig. $3 \mathrm{~b}$ the corresponding trajectory is shown, which leads to the equilibrium $S_{1}$ where predators are extinct, according to Proposition 2. Similarly, if the Fishing Authority decides to give 25 licences for fishing the prey only and 25 licences for fishing the predator only, i.e. $N=50$ and $r=0.5$ (fixed) for preventing overexploitation, then the system converges to the predators extinction equilibrium $S_{1}^{r}$, as determined in Proposition 3 and shown in Fig. 3c. Of course the value of $r$ in this numerical simulation is not optimally chosen by solving a suitable optimal control problem, but we just assumed the rough rule of thumb of dividing the fishermen into two groups of equal number. Instead, Figs. 3d,e show the time evolutions of preys and predators when the parameter $r$ is not fixed but it is endogenously chosen by fishermen on the basis of the profit-driven evolutionary mechanism in continuous time and discrete time respectively, as described in section 4 . It is worth specifying that Figs. 3d,e represent the projection on the two-dimensional space $\left(X_{1}, X_{2}\right)$ of trajectories generated by three-dimensional dynamical systems where the third dynamical variable $r(t)$ is modeled with a discrete switching time $s=3$ in Fig. 3e, and continuous time evolution, i.e. $s \rightarrow 0$, in Fig. 3d. The two trajectories exhibit a similar asymptotic behavior, even if their transient portions are different. Indeed, they converge to the same biological coexistence equilibrium $S_{5}^{e}$ (see Proposition 4), with the same final share of agents fishing species 1 , given by $r \simeq 0.664564$. However, in the discrete case the dynamic is characterized by "jumps", which are evident in Fig. 4f, where the evolution of $r(t)$ is shown versus time along the trajectory of Fig. 3e (compare Figs. 4e,f). This example confirms that for some parameter 


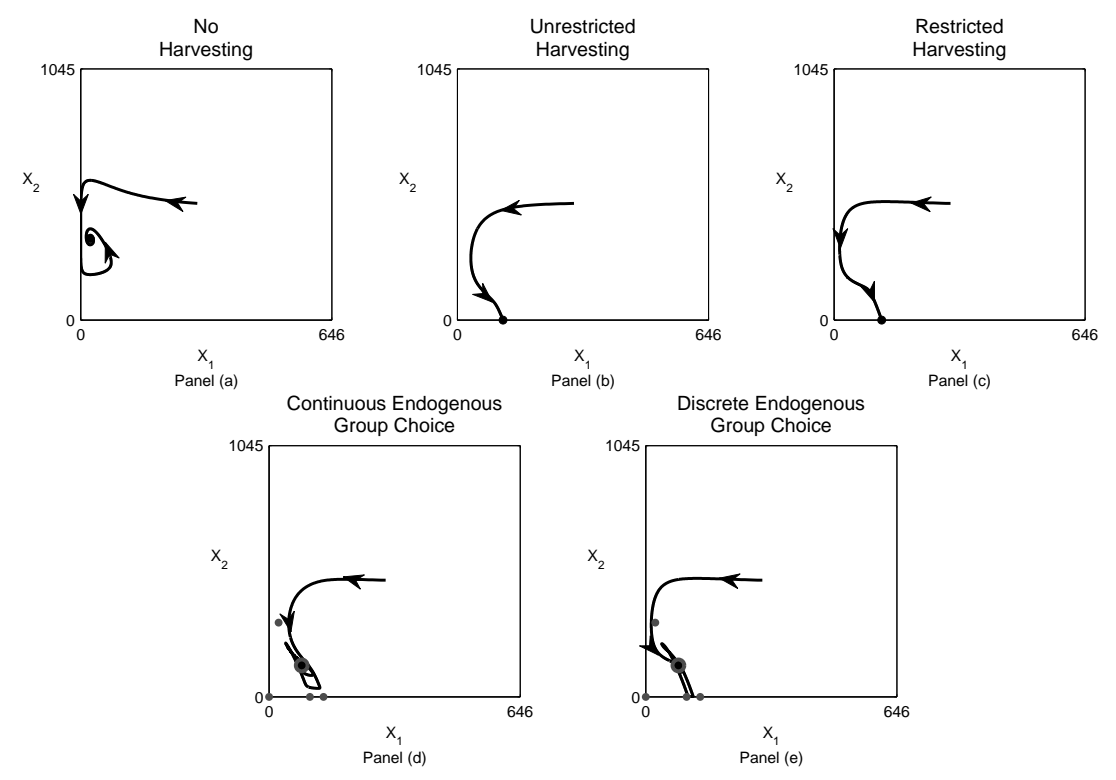

Figure 3: Trajectories in the phase space $\left(X_{1}, X_{2}\right)$ with parameters as in Fig. 1 and $k=140, \eta=0.6$, $\gamma_{1}=4, \gamma_{2}=6.2, N=50, a_{1}=a_{2}=10, b_{1}=b_{2}=0$ and initial condition $X_{1}(0)=300, X_{2}(0)=485$, $r(0)=0.5$. (a) Rosenzweig-MacArthur prey-predator model without harvesting. (b) Unrestricted harvesting. (c) Restricted harvesting with imposed $r=0.5$. (d) Endogenous $r(t)$ in continuous time. (e) Hybrid model with $r(t)$ in discrete time. In all the figures gray points represent unstable equilibria and gray points with a hole represent stable equilibria for the continuous evolutionary model.

settings the model with continuous-time switching may provide a good benchmark for understanding the dynamical properties of the more realistic, but also more involved, hybrid system. In both cases the state variable $r$ converges to the same equilibrium value, with the only difference in the speed of convergence, which is much higher in the continuous switching case. For the fishermen this means less profits in case of discrete adjustment mechanism during the initial transient. However, in the two examples the same biomass of preys and predators as well as the same profits are obtained in the long run. Figs. 4a,b,c,d, where the time evolutions of total profits computed along the same trajectories of Figs. 3b,c,d,e are represented, show that the model with the endogenous adaptive switching mechanism could also exhibit good performances.

In this specific example, the highest profits are obtained under unrestricted harvesting, so that unrestricted harvesting would seam to be a good practice for the fishermen. However, here unrestricted harvesting leads to overexploitation, as it reduces the carrying capacity of the prey so that predators become extinct, see again Fig. 3b. On the contrary, the endogenous pulse switching mechanism is able to ensure a good compromise between profits and sustainable exploitation of both species.

In Fig. 5, we increase the value of the carrying capacity to $K=600$, so that the model without harvesting presents persistent oscillations along a stable limit cycle, as described in Section 3.1 (see Fig. 5a). This means that the prey-predator ecosystem is characterized by oversupply of nutrients 

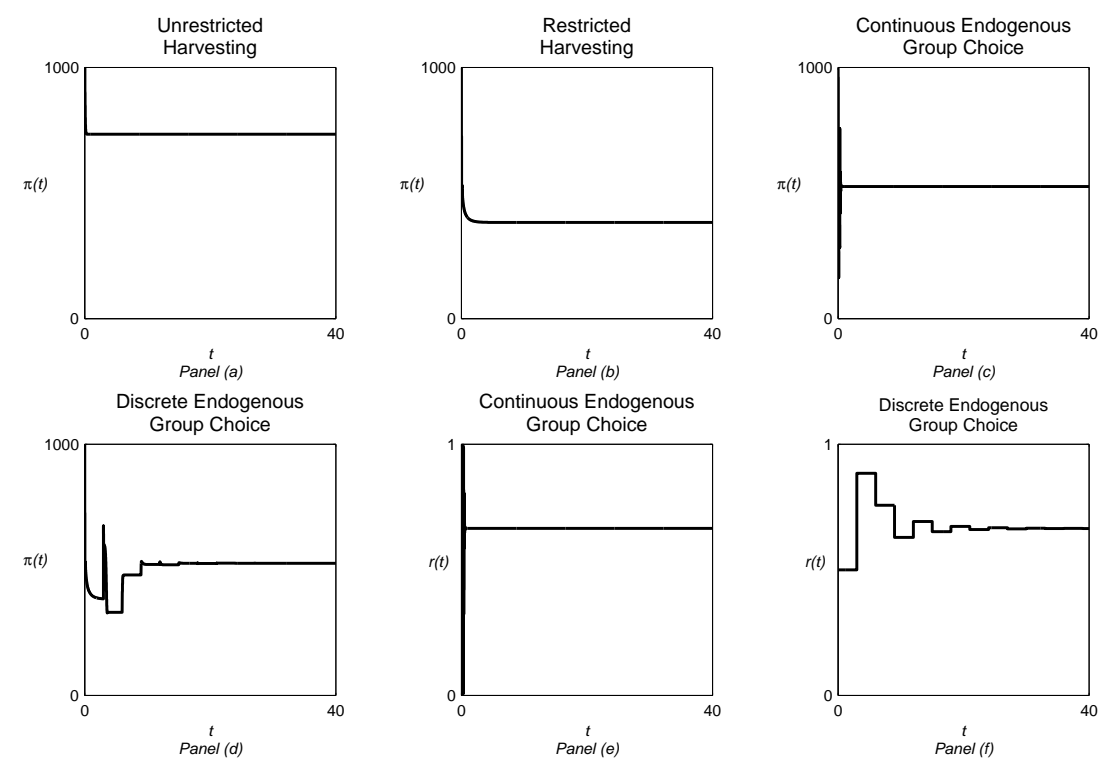

Figure 4: Versus-time representation of total profits along the trajectories of the model with: (a) Unrestricted harvesting; (b) Restricted harvesting with imposed $r=0.5$; (c) Endogenous $r(t)$ in continuous time; (d) Hybrid model with $r(t)$ in discrete time. Versus time evolution of $r(t)$ in: (e) continuous time. (f) discrete time (all parameters as in Fig. 3). 


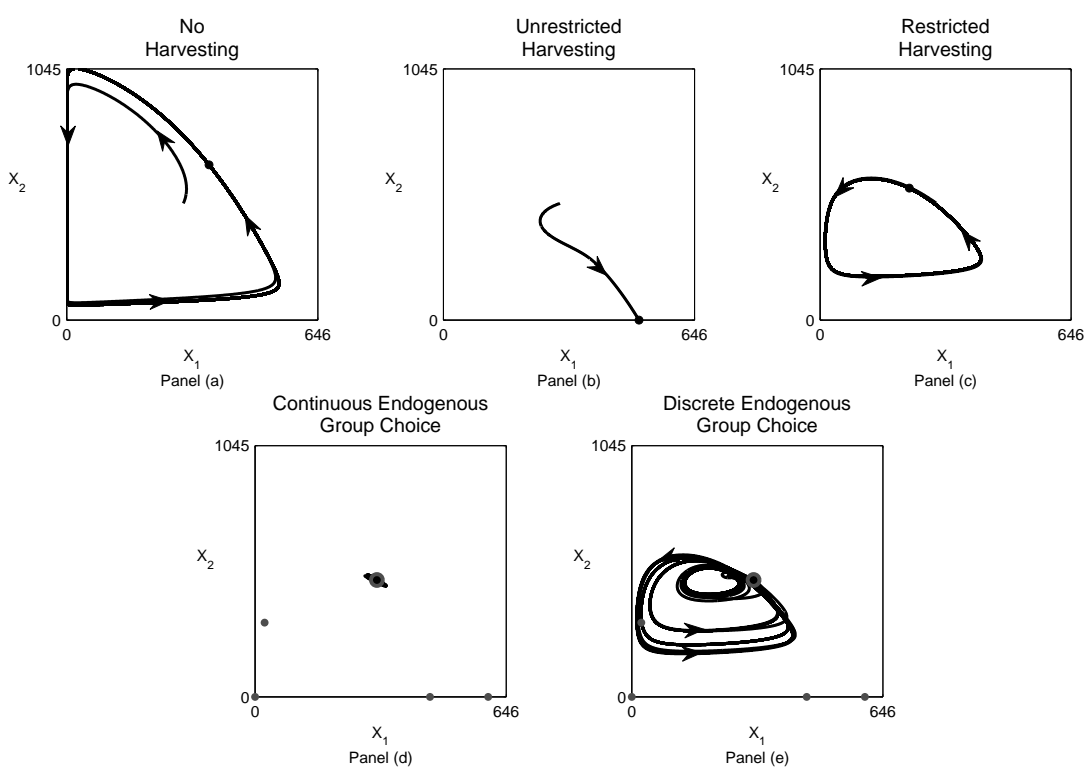

Figure 5: Trajectories in the phase space $\left(X_{1}, X_{2}\right)$ with initial condition and parameters as in Fig. 3 but $K=600$. (a) Rosenzweig-MacArthur prey-predator model without harvesting. (b) Unrestricted harvesting. (c) Restricted harvesting with imposed $r=0.5$. (d) Endogenous $r(t)$ in continuous time. (e) Hybrid model with $r(t)$ in discrete time.

at the bottom of the food chain that leads to persistent oscillations (according to the "paradox of enrichment" see e.g. [34], [27], [35], [36]). In the long run, the model with unrestricted harvesting (Fig. $5 \mathrm{~b}$ ) leads to predators' extinction and with imposed $r=0.5$ (Fig. 5c) it has persistent oscillations. On the contrary, with the same initial conditions and parameter values, both models with endogenous switching in continuous time (Fig. 5d) and in discrete time (Fig. 5e) converge to a stable equilibrium where preys and predators coexist in the stationary state denoted by $S_{5}^{e}$ in Proposition 4 . We notice that in this case the evolutionary model with endogenous switching helps to stabilize the preyspredators coexistence equilibrium, i.e. it helps avoiding the paradox of enrichment. Therefore, from a practical point of view, while the definition of an optimal value of $r$ is not an easy task, as it requires time, money and farsightedness, the evolutionary switching mechanism described in this paper seems to bring good results, although exploiters are allowed to adopt short-run optimizing strategies, which would lead to overexploitation or extinction when totally unregulated.

The simulations depicted in Fig. 6 are obtained with $K=600$ and the other parameter as before. The initial condition of the system is taken sufficiently close to the inner equilibrium $S_{4}^{e}$. According to Proposition 4 , the border equilibrium $S_{4}^{e}=\left(\widetilde{X}_{1}^{e}, \widetilde{X}_{2}^{e}, 1\right)$ has already lost its stability through a supercritical Hopf bifurcation since $K>K_{H}=\frac{2 \gamma_{1} \alpha(d+\rho \eta \beta)}{\left(2 \gamma_{1} \rho-N a_{1}\right)(\eta \beta-d)} \simeq 219.74$, being $K_{H}$ the Hopf bifurcation curve for that equilibrium, according to Proposition4. It follows that for suitable initial conditions, the system with replicator dynamics in continuous time (27) converges to a stable limit cycle, see Fig. 6d. Therefore, in this case the model in continuous time admits the coexistence of two 

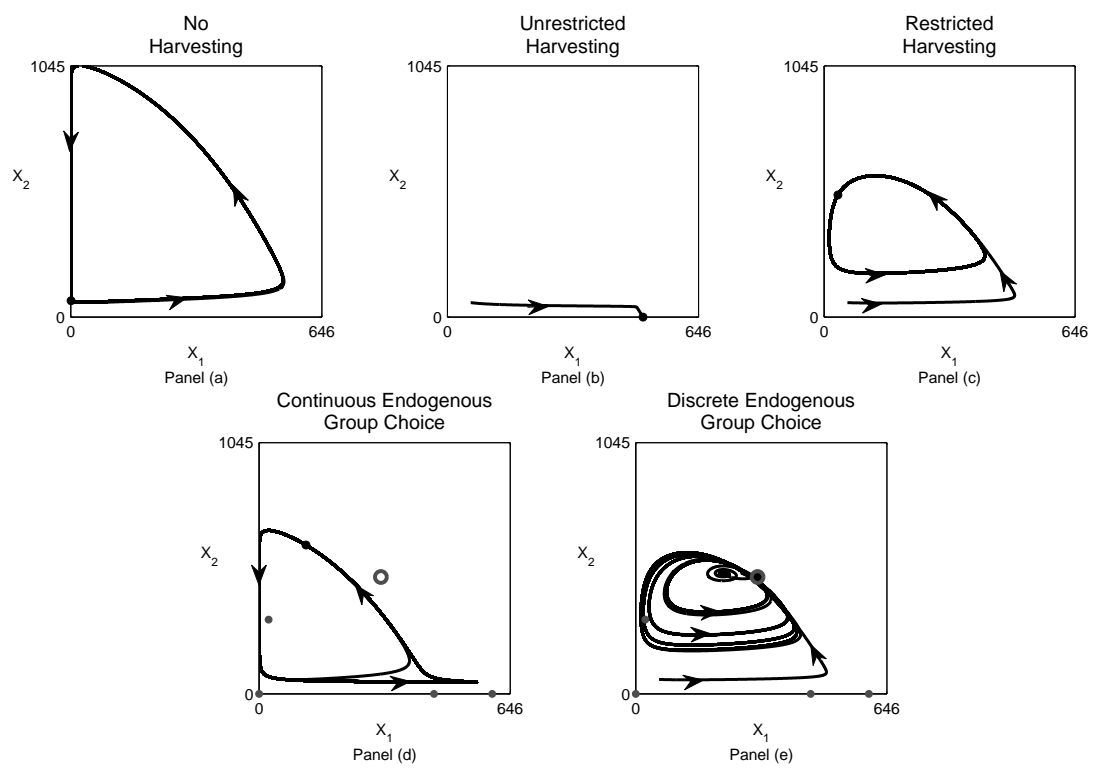

Figure 6: Trajectories in the phase space $\left(X_{1}, X_{2}\right)$ with parameters as in Fig. 5 but initial condition $X_{1}(0)=60, X_{2}(0)=60, r(0)=0.5$ (a) Rosenzweig-MacArthur prey-predator model without harvesting. (b) Unrestricted harvesting. (c) Restricted harvesting with imposed $r=0.5$. (d) Endogenous $r(t)$ in continuous time. (e) Hybrid model with $r(t)$ in discrete time.

stable attractors, the stable steady state $S_{5}^{e}$ and the stable limit cycle bifurcating from $S_{4}^{e}$. However, in the hybrid case we always detected the convergence to the inner equilibrium, no matter what the initial condition is. It proves that in some cases the presence of pulse dynamics could stabilize the system. This stabilizing effect can also be stressed through the inspection of the basins of attraction, shown in Fig. 7. In Fig. 7a the white region represents the basin of attraction of equilibrium $S_{5}^{e}$ and the black region is the basin of attraction of the limit cycle depicted in Fig. 6d. For the hybrid system, the generic trajectory with initial condition in the square $\left(X_{1}, X_{2}\right) \in(0.1,600) \times(0.1,600)$ always converges to the inner equilibrium $S_{5}^{e}$. With respect to the third dynamic variable, all the basins here shown are obtained with initial condition $r=0.5$. However, other simulations not reported here show similar scenarios also for different initial values of $r$.

With all parameters as in Fig. 6, except $K=650$, we obtain the example shown in Fig. 8. In Fig. 8a two coexisting stable limit cycles are created through supercritical Hopf bifurcations of $S_{4}^{e}$ (black curve) and $S_{5}^{e}$ (gray curve) in the model with continuous replicator dynamics (27). Notice that no stable equilibrium exists in this case for the system (27) according to Proposition 4. This case gives us the opportunity to discuss some similarities and differences between the continuous and the hybrid model. So far, the numerical analysis has shown that the dynamics of the hybrid model converged to the inner equilibrium whenever $S_{5}^{e}$ was locally asymptotically stable for the evolutionary system in continuous time. In addition, Fig. $8 \mathrm{~b}$ shows that the stability of the inner equilibrium $S_{5}^{e}$ in the hybrid model may hold even when it is not a stable in the model with continuous time switching (27). In 

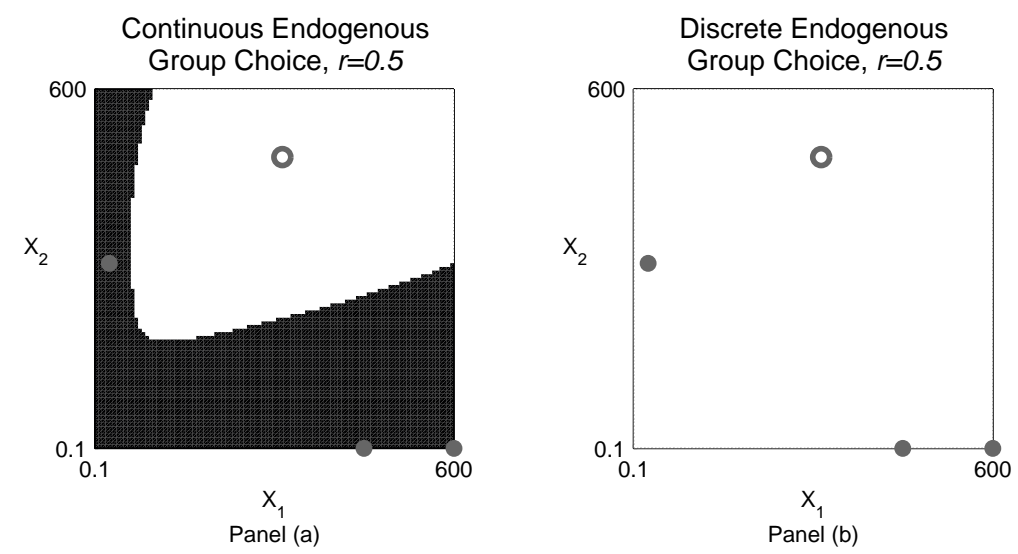

Figure 7: Basin of attractions with initial conditions $\left(X_{1}(0), X_{2}(0)\right)$ in the square $(0.1,600) \times(0.1,600)$ and with initial $r=0.5$ for the model with endogenous $r(t)$ in: (a) continuous time. (b) discrete time. Parameters as in Figs. 5 and 6. White region is the basin of attraction of the inner equilibrium $S_{5}^{e}$; Black region is the basin of attraction of the stable closed invariant orbit in Fig. 6d. 

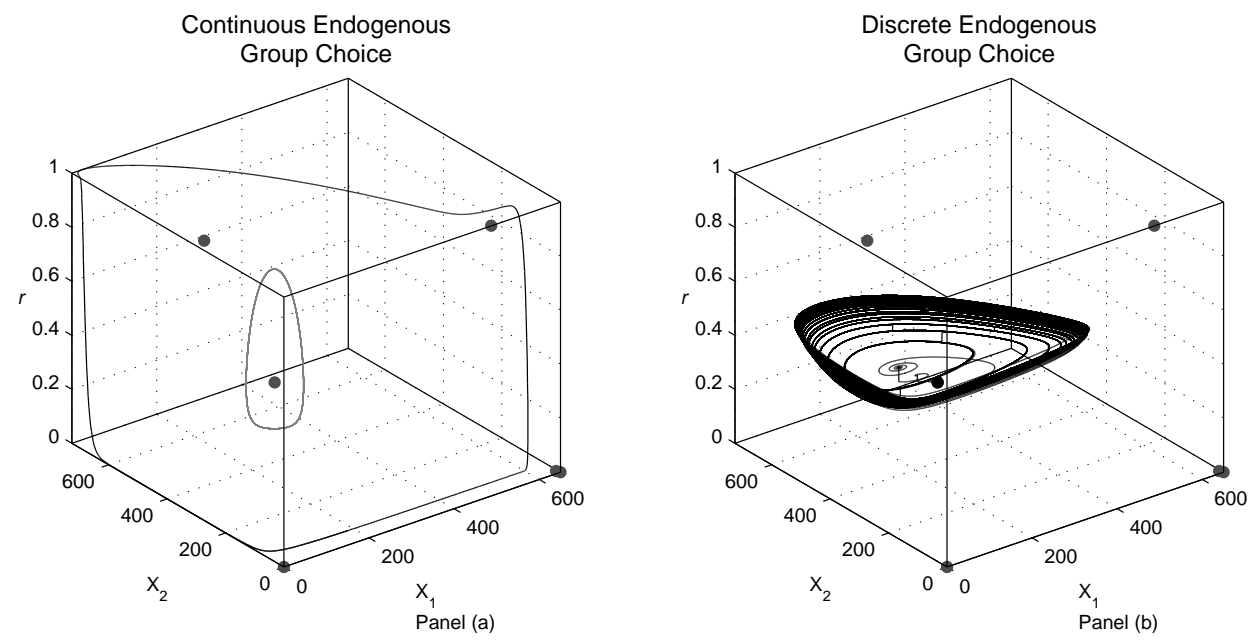

Figure 8: Trajectories in the phase space $\left(X_{1}, X_{2}, r\right)$ with parameters as in Fig. 6 but $K=650$. (a) Endogenous $r(t)$ evolving according to a continuous time replicator dynamics. The stable gray orbit appears through a supercritical Hopf bifurcation of $S_{5}^{e}$; the stable black one appears through a supercritical Hopf bifurcation of $S_{4}^{e}$. (b) Hybrid model with $r(t)$ in discrete time.

Fig. 8a the trajectory of the model with continuous switching is plotted without a transient to better emphasize the two stable limit cycles. The two initial conditions taken in the basins of attraction of the two different limit cycles are, respectively, $X_{1}(0)=300, X_{2}(0)=485, r(0)=0.5$ and $X_{1}(0)=60$, $X_{2}(0)=60, r(0)=0.5$. In Fig. 8 b, the whole trajectory (i.e. with the transitory part) of the hybrid dynamical system is plotted. ${ }^{2}$

Another way to compare the different dynamical systems is the numerical study of the twoparameters bifurcation diagram in the space $(\eta, K){ }^{3}$ In Fig. 9a,b we show these diagrams for the cases of continuous and discrete evolutionary dynamics. The parameter constellation is the same as in Fig. 1a and Figs. 2a,c, so that a direct comparison can be carried out ${ }^{4}$. The two-parameters bifurcation diagrams in Fig. 2 and Fig. 9 emphasize that in all the considered dynamical systems, there are three possible long-run behaviors: 1) convergence to a stable border equilibrium, characterized by predator extinction or one-species harvesting (grey region); 2) convergence to a stable inner equilibrium, characterized by coexistence and harvesting of both species (white region); and 3) convergence to an attractor with persistent oscillations dynamics, characterized by coexistence and harvesting of the two species (black region). The bifurcation diagrams give numerical evidence that the dynamical systems without harvesting and the one with evolutionary switching have several analogies. Indeed, the transcritical bifurcation curves, marking the transition from grey to white areas, look very similar for these

\footnotetext{
${ }^{2}$ For graphical reasons in Fig. 8b we have only shown the trajectory starting from $X_{1}=300, X_{2}=485, r=0.5$, although also the trajectory with the other initial condition converges to the inner equilibrium.

${ }^{3}$ The choice of $K$ as bifurcation parameter is standard for the Rosenzweig-MacArthur model (see e.g. [27]) while $\eta$ is chosen for convenience. The same analysis with other parameters may also be useful, but it would lead to quite similar results.

${ }^{4}$ Notice that, apart from the bifurcation parameters $\eta$ and $K$, the remaining parameters are fixed as in Fig. 3. The same set of parameters is employed also in all the other figures of this paper, with the exception of Fig. 10 where $b_{1,2} \neq 0$.
} 

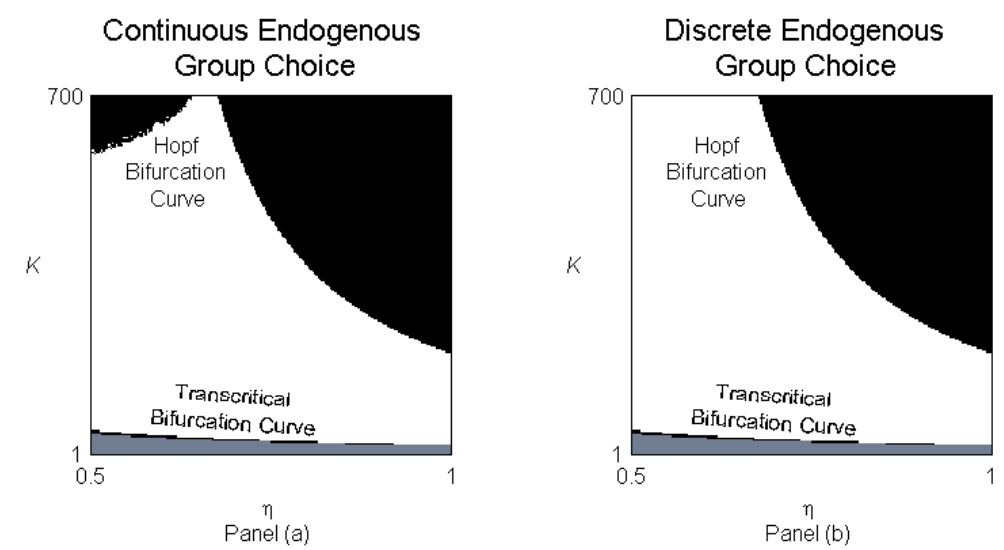

Figure 9: Bifurcation diagrams in the parameters space $(\eta, K) \in(0.5,1) \times(1,700)$ : white region represents couple of parameters such that the system converges to the stable inner equilibrium $S_{5}^{e}$; for parameters in the black region there is persistent cyclic behavior along a stable limit cycle around $S_{5}^{e}$; in the gray areas $S_{5}^{e}$ is not feasible. (a) continuous replicator dynamics. (b) Hybrid model.

two models. This means that, if there are suitable ecological conditions for the stable coexistence of the two stocks, then it is highly probable that these conditions also ensure the coexistence in case of harvesting with evolutionary switching. Moreover, from the bifurcation diagrams, it is clear that persistent oscillations are more common for the natural model without harvesting than in the evolutionary model, because the region of stationary coexistence (i.e. stability of the positive equilibrium) is larger for the model with harvesting under evolutionary switching. In other words, the evolutionary fishery mechanism modeled in this paper can even enhance stability in cases where the unexploited resource exhibits persistent oscillatory dynamics, as it may reduce the destabilizations caused by an excess of nutrients available to the preys, i.e. an increase of $K$. Notice that, in the case of unrestricted harvesting (Fig. 2a), the grey region extends over almost the entire parameter space, thus leading to a low probability that predators will survive in the long run, much lower than in the other scenarios, according to the paradigm of the tragedy of the commons. The two parameters bifurcation diagrams of Fig. 9 also suggest that, in general, the two proposed evolutionary models have different stability regions. On the contrary to what one would expect, the pulse dynamics model may have a stabilizing effect. In fact, in the case under consideration, there are pairs of parameter values $(K, \eta)$ for which the inner equilibrium is unstable for the continuous time evolutionary model and stable for the discrete time evolutionary model. In this particular case, these pairs are located near the left upper corners of Figs. 9a,b. Notice that this is precisely what we have already observed in the numerical simulations shown in Fig. 8.

Up to now, we only considered cases with perfectly elastic demand for the two species. In the following example we relax this assumption in order to understand the possible effect of non-constant prices in the dynamics of the models. For the sake of comparison, all the parameters are set as in Fig. 


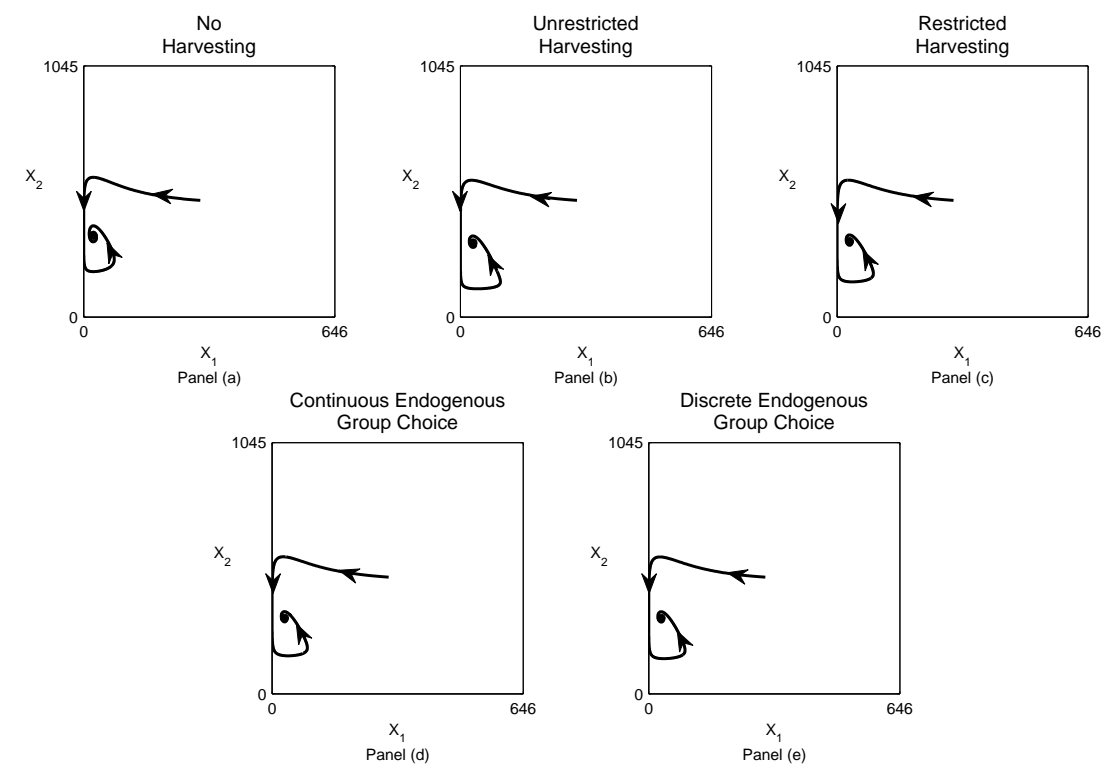

Figure 10: Trajectories in the phase space $\left(X_{1}, X_{2}\right)$ with initial condition and parameters as in Fig. 3 but $b_{1}=b_{2}=0.01$ and $\sigma=1 / 2$ (a) Rosenzweig-MacArthur prey-predator model without harvesting. (b) Unrestricted harvesting. (c) Restricted harvesting with imposed $r=0.5$. (d) Endogenous $r(t)$ with continuous time replicator dynamics. (e) Hybrid model.

3 , but $b=0.01$. The different dynamic behaviors are evident by comparing Figs. 3 and 10. In this case, the higher is the quantity of fish in the market, the lower is its selling price, so that this effect reduces the overexploitation and the long-run dynamics settle to an inner equilibrium in all the cases.

In conclusion, the hybrid model exhibits in most cases convergence to the inner equilibrium, despite a strange transient dynamics. However, also attractors different from fixed points can be present, as indicated in the two parameters bifurcation diagram of Fig. 9b. A plausible explanation of the stabilizing effect observed in the numerical simulations is based on the role played by $s$, i.e. the length of time after which fishermen are allowed to change their harvesting strategies according to past profits. As $s \rightarrow 0$, the hybrid model tends to the continuous one and fishermen react immediately to changes in instantaneous harvesting strategy profits. As $s$ increases the fishermen decisions occur with a higher degree of inertia. Moreover, they base their decision upon a more sophisticated timestructure information about past profits, i.e. mobile time averages of profits observed in the past, and this has a stabilizing role as well.

\section{Some conclusions and further developments}

In this paper a hybrid dynamical system is proposed to model a fishery where two species in preypredator relationship are harvested by a population of fishermen who are allowed to catch only one of the two species at a time, and to change the caught variety at discrete time pulses, according to a profit- 
driven replicator dynamics. However, the dynamic equations describing the growth and interaction of the two fish species are always in continuous time. The analytical and numerical results show that this type of evolutionary mechanism may lead to a good compromise between profit maximization and resource conservation thanks to an evolutionary self-regulation based on cost and price externalities. In fact, the reduction of biomass of one species leads to increasing landing costs and it consequently favours the endogenous switching to the more abundant species. Moreover, severe overfishing of one species causes decreasing prices and consequently decreasing profits.

The employed prey-predator model, namely logistic growth and Holling type II function response, is simple and widely employed in the literature. Nevertheless, introducing harvesting with impulsive evolutionary switching in discrete time makes the model quite complicated to be studied analytically. For this reason, some simpler benchmark cases, with fixed prices or continuous time switching, have also been developed here. Although these benchmarks may seem quite unrealistic, they constitute a useful guide, even a sort of basic foundation on which the (mainly numerical) analysis of the more realistic model with variable market prices and impulsive strategy switching can be built upon.

In the paper we have carried out several comparisons between continuous time and discrete time (or impulsive) switching according to the profit-driven replicator dynamics. Our numerical results show that in some cases the region of stability of the inner equilibrium is larger in the hybrid system than in the continuous-time model. Other remarkable features of the hybrid system are related to the possibility of reducing long run oscillation dynamics as well as to avoiding the occurrence of bistability. This seems to be in contrast with some results in the literature stressing the fact that discrete replicator dynamics generated oscillatory behaviors (see e.g. [9]). However in our case we have a hybrid model where the discrete replicator switching is embedded in an underlying model in continuous time. Moreover, the switching is decided according to a moving average of profits, and this has a stabilizing effects because it introduces a form of inertia.

From the point of view of population dynamics, the endogenous switching mechanism, in which fishermen decide the variety to catch on the basis of their profits, attenuates some negative effects of unrestricted harvesting. In fact, in some cases if the dynamics of the unexploited species converge to the stable coexistence equilibrium, then it is highly probable that coexistence is achieved with harvesting strategy switching (in continuous or discrete time), thus significantly reducing the negative effects of exploitation. Another surprising characteristic of this endogenous switching is the reduction of the "oscillatory effect" due to oversupply of food. In fact, it is well known that, in a food-chain population model, the presence of self-sustained oscillations means oversupply of nutrients. In [27] some practical rules are given to reduce oscillations caused by overabundance of food at the bottom of the food chain.

The exercise carried out here offers glimpse into the interesting properties of myopic and adaptive harvesting mechanisms driven by endogenous evolutionary processes. However this is just a starting point for further and deeper analysis. There are several aspects of the model that deserve to be explored more deeply. For example, the variable $r$, i.e. the fraction of fishermen harvesting a given fish stock, is assumed to unconstrainedly range in the interval [0,1], where 0 and 1 are always equilibria. When $r$ converges to 0 or 1 , one of the two species is no longer harvested and consequently it is not available in the market. This could be an acceptable outcome only if the two species of fish are perfect 
substitute in consumers tastes (corresponding to the case $\sigma=1 \mathrm{in}$ our model). Otherwise consumers may be heavily penalized by such equilibrium strategies. This issue will be addressed in future work, for example by introducing constraints on the dynamics of $r$. The research could be extended in other different directions as well. First of all, it would be interesting to compare the results obtained here with those where an optimal fraction $r$ is computed according to an optimal control problem, in which a social welfare function is maximized over time. Moreover, the stability analysis for the model with continuous evolutionary switching mechanism may be extended to provide indications on the behavior of the hybrid dynamical system in the long run.

\section{Appendix A}

\subsection{Proof of Proposition 2}

To investigate the stability properties of the equilibria by linearization, we consider the Jacobian matrix of (19):

$$
J=\left[\begin{array}{cc}
\rho-\frac{2 \rho X_{1}}{K}-\frac{\alpha \beta X_{2}}{\left(\alpha+X_{1}\right)^{2}}-\frac{N a_{1}}{2 \gamma_{1}} & -\frac{\beta X_{1}}{\alpha+X_{1}} \\
\frac{\eta \beta \alpha X_{2}}{\left(\alpha+X_{1}\right)^{2}} & \frac{\eta \beta X_{1}}{\alpha+X_{1}}-d-\frac{a_{2} N}{2 \gamma_{2}}
\end{array}\right]
$$

At the extinction equilibrium $S_{0}$ the Jacobian matrix is diagonal:

$$
J\left(S_{0}\right)=\left[\begin{array}{cc}
\rho-\frac{N a_{1}}{2 \gamma_{1}} & 0 \\
0 & -d-\frac{a_{2} N}{2 \gamma_{2}}
\end{array}\right]
$$

with eigenvalues $\lambda_{1}=\rho-\frac{N a_{1}}{2 \gamma_{1}}$ and $\lambda_{2}=-d-\frac{a_{2} N}{2 \gamma_{2}}<0$. Therefore $S_{0}$ is a stable node for $2 \gamma_{1} \rho<N a_{1}$, i.e. when the total fishing effort level exceeds the intrinsic growth rate of the prey population. Instead, $S_{0}$ is a saddle point, and $S_{1}$ becomes positive (through a transcritical bifurcation) when $2 \rho \gamma_{1}>N a_{1}$. From the triangular structure of the Jacobian matrix in $S_{1}=\left(\bar{X}_{1}, 0\right)$

$$
J\left(S_{1}\right)=\left[\begin{array}{cc}
-\rho+\frac{N a_{1}}{2 \gamma_{1}} & -\frac{\beta \overline{X_{1}}}{\alpha+\overline{X_{1}}} \\
0 & \frac{\eta \beta K\left(2 \rho \gamma_{1}-N a_{1}\right)}{\alpha 2 \rho \gamma_{1}+K\left(2 \rho \gamma_{1}-N a_{1}\right)}-d-\frac{a_{2} N}{2 \gamma_{2}}
\end{array}\right]
$$

it is easy to see that $S_{1}$ is a stable node when $\rho>\frac{N a_{1}}{2 \gamma_{1}}$ and $\frac{\eta \beta K\left(2 \rho \gamma_{1}-N a_{1}\right)}{\alpha 2 \rho \gamma_{1}+K\left(2 \rho \gamma_{1}-N a_{1}\right)}-d-\frac{a_{2} N}{2 \gamma_{2}}<0$. Instead, when the interior equilibrium $S_{2}$ enters the positive orthant the boundary equilibrium $S_{1}$ becomes a saddle, with stable manifold along the $X_{1}$ axis and unstable manifold transverse to it, via transcritical bifurcation.

The Jacobian of the system in $S_{2}$ is:

$$
J\left(S_{2}\right)=\left[\begin{array}{cc}
\frac{\left(d+N \frac{a_{2}}{2 \gamma_{2}}\right)\left(\left(\rho 2 \gamma_{1}-N a_{1}\right) K\left(\eta \beta-d-N \frac{a_{2}}{2 \gamma_{2}}\right)-2 \rho \gamma_{1} \alpha\left(\eta \beta+d+N \frac{a_{2}}{2 \gamma_{2}}\right)\right)}{2 K \gamma_{1} \eta \beta\left(\eta \beta-d-N \frac{a_{2}}{2 \gamma_{2}}\right)} & -\frac{\beta X_{1}^{*}}{\alpha+X_{1}^{*}} \\
\frac{\eta \beta \alpha X_{2}^{*}}{\left(\alpha+X_{1}^{*}\right)^{2}} & 0
\end{array}\right]
$$

When $\left(2 \rho \gamma_{1}-N a_{1}\right) K\left(\eta \beta-d-N \frac{a_{2}}{2 \gamma_{2}}\right)-2 \rho \gamma_{1} \alpha\left(d+N \frac{a_{2}}{2 \gamma_{2}}\right)$ decreases across zero, $S_{2}$ merges with $S_{1}$ and then it exits the positive orthant, and $S_{1}$ becomes stable through a transcritical bifurcation. Instead, if $\left(\rho 2 \gamma_{1}-N a_{1}\right) K\left(\eta \beta-d-N \frac{a_{2}}{2 \gamma_{2}}\right)-2 \rho \gamma_{1} \alpha\left(\eta \beta+d+N \frac{a_{2}}{2 \gamma_{2}}\right)<0$ the equilibrium is stable, 
while, when this inequality is reversed, it becomes an unstable focus through a supercritical Hopf bifurcation $^{5}$ after which an attractive limit cycle appears around it.

\subsection{Proof of Proposition 3}

The Jacobian matrix of (22)

$$
J=\left[\begin{array}{cc}
\rho-\frac{2 \rho X_{1}}{K}-\frac{\alpha \beta X_{2}}{\left(\alpha+X_{1}\right)^{2}}-r \frac{N a_{1}}{2 \gamma_{1}} & -\frac{\beta X_{1}}{\alpha+X_{1}} \\
\frac{\eta \beta \alpha X_{2}}{\left(\alpha+X_{1}\right)^{2}} & \frac{\eta \beta X_{1}}{\alpha+X_{1}}-d-(1-r) \frac{a_{2} N}{2 \gamma_{2}}
\end{array}\right]
$$

computed at the global extinction equilibrium $S_{0}=(0,0)$ becomes

$$
J\left(S_{0}\right)=\left[\begin{array}{cc}
\rho-r \frac{N a_{1}}{2 \gamma_{1}} & 0 \\
0 & -d-(1-r) \frac{a_{2} N}{2 \gamma_{2}}
\end{array}\right]
$$

so the eigenvalues are both negative if $2 \gamma_{1} \rho<r N a_{1}$. If $2 \rho \gamma_{1}>r N a_{1}$ then $S_{0}$ is a saddle point and $S_{1}^{r}$ is positive. From

$$
J\left(S_{1}^{r}\right)=\left[\begin{array}{cc}
-\rho+r \frac{N a_{1}}{2 \gamma_{1}} & -\frac{\beta \overline{X_{1}}}{\alpha+\overline{X_{1}}} \\
0 & \frac{\eta \beta K\left(2 \rho \gamma_{1}-N a_{1}\right)}{\alpha 2 \rho \gamma_{1}+K\left(2 \rho \gamma_{1}-N a_{1}\right)}-d-(1-r) \frac{a_{2} N}{2 \gamma_{2}}
\end{array}\right]
$$

it is plain to see that $S_{1}^{r}$ is a stable node whenever the elements in the principal diagonal of $J\left(S_{1}^{r}\right)$ are negative.

If the interior equilibrium $S_{2}^{r}$ is positive, then the boundary equilibrium $S_{1}^{r}$ is a saddle. From

$$
J\left(S_{2}^{r}\right)=\left[\begin{array}{cc}
\frac{\left(d+(1-r) N \frac{a_{2}}{2 \gamma_{2}}\right)\left[\left(2 \gamma_{1} \rho-r N a_{1}\right) K\left(\eta \beta-d-(1-r) N \frac{a_{2}}{2 \gamma_{2}}\right)-2 \gamma_{1} \rho \eta \beta \alpha\right]}{2 \gamma_{1} K \eta \beta\left(\eta \beta-d-(1-r) N \frac{a_{2}}{2 \gamma_{2}}\right)} & -\frac{\beta X_{1}^{i *}}{\alpha+X_{1}^{i *}} \\
\frac{\eta \beta \alpha X_{2}^{i *}}{\left(\alpha+X_{1}^{i *}\right)^{2}} & 0
\end{array}\right]
$$

it is easy to see that $S_{2}^{r}$ is stable for $\left(2 \gamma_{1} \rho-r N a_{1}\right) K\left(\eta \beta-d-(1-r) N \frac{a_{2}}{2 \gamma_{2}}\right)-2 \gamma_{1} \rho \alpha\left(\eta \beta+d+(1-r) N \frac{a_{2}}{2 \gamma_{2}}\right)$ 0 and unstable otherwise, with stability loss occurring via a supercritical Hopf bifurcation, as it can be seen numerically (see footnote at the end of the proof of Proposition 2).

It is worth noticing that for $\left(\eta \beta-d-(1-r) \frac{a_{2} N}{2 \gamma_{2}}\right) K\left(2 \rho \gamma_{1}-N a_{1}\right)-2 \rho \gamma_{1} \alpha\left(d+(1-r) \frac{a_{2} N}{2 \gamma_{2}}\right)=0$, $S_{2}^{r}$ merges with $S_{1}^{r}$ and when the left hand side is negative the equilibrium $S_{2}^{r}$ is no longer in the positive orthant and the equilibrium $S_{1}^{r}$ becomes stable through a transcritical bifurcation.

\section{Appendix B. Proof of Proposition 4}

\section{Existence of equilibria.}

Equilibrium points are the solutions of the algebraic system

\footnotetext{
${ }^{5} \mathrm{~A}$ rigorous proof of the supercritical or subcritical nature of Hopf bifurcation requires a center manifold reduction and the evaluation of higher order derivatives, up to the third order (see e.g. [37]). This is rather tedious in a two-dimensional system, and we claim numerical evidence in order to ascertain the nature of such bifurcations.
} 


$$
\begin{aligned}
X_{1}\left[\rho\left(1-\frac{X_{1}}{K}\right)-\frac{\beta X_{2}}{\alpha+X_{1}}-r N \frac{a_{1}}{2 \gamma_{1}}\right] & =0 \\
X_{2}\left[\frac{\eta \beta X_{1}}{\alpha+X_{1}}-d-(1-r) N \frac{a_{2}}{2 \gamma_{2}}\right] & =0 \\
r(1-r)\left(\frac{a_{1}^{2}}{4 \gamma_{1}} X_{1}-\frac{a_{2}^{2}}{4 \gamma_{2}} X_{2}\right) & =0
\end{aligned}
$$

from which it is straightforward to obtain the equilibria $S_{0}^{e}, S_{1}^{e}, S_{2}^{e}, S_{3}^{e}, S_{4}^{e}$.

As for the equilibrium $S_{5}^{e}$, when $r \in(0,1)$, the third equation in (29) is satisfied when $X_{2}=\frac{\gamma_{2} a_{1}^{2}}{\gamma_{1} a_{2}^{2}} X_{1}$ so that the first and second equations become

$$
\begin{aligned}
\frac{X_{1}}{\alpha+X_{1}}\left[\left(\rho-\frac{\rho X_{1}}{K}\right)\left(\alpha+X_{1}\right)-\frac{\beta a_{1}^{2} \gamma_{2} X_{1}}{a_{2}^{2} \gamma_{1}}-\frac{r N a_{1}}{2 \gamma_{1}}\left(\alpha+X_{1}\right)\right] & =0 \\
\frac{\gamma_{2} a_{1}^{2}}{\gamma_{1} a_{2}^{2}} X_{1}\left(\frac{\eta \beta X_{1}}{\alpha+X_{1}}-d-(1-r) \frac{N a_{2}}{2 \gamma_{2}}\right) & =0
\end{aligned}
$$

From the second one we have $X_{1}(r)=\frac{\alpha\left(2 d \gamma_{2}+(1-r) N a_{2}\right)}{2 \eta \beta \gamma_{2}-2 d \gamma_{2}-(1-r) N a_{2}}$, so that the first equation in (29) can be written as

$$
\frac{\alpha\left(N a_{2}(1-r)+2 d \gamma_{2}\right)}{2 a_{2}^{2} K \gamma_{1} \eta\left(-a_{2} N(1-r)-2 \gamma_{2}(d-\beta \eta)\right)^{2}}\left[A r^{2}+B r+C\right]=0,
$$

with

$$
\begin{aligned}
& A=a_{1} a_{2}^{2} K N^{2}\left(a_{1}-a_{2} \eta\right) \\
& B=a_{2} N\left\{a_{1} K\left\{-2 a_{1}\left(a_{2} N+2 d \gamma_{2}-\beta \gamma_{2} \eta\right)+a_{2} \eta\left[a_{2} N+2 \gamma_{2}(d-\beta \eta)\right]\right\}+2 a_{2}^{2}(K+\alpha) \gamma_{1} \eta \rho\right\} \\
& C=a_{1}^{2} K\left(a_{2} N+2 d \gamma_{2}\right)\left[a_{2} N+2 \gamma_{2}(d-\beta \eta)\right]-2 a_{2}^{2} \gamma_{1} \eta\left[(K+\alpha)\left(a_{2} N+2 d \gamma_{2}\right)-2 K \beta \gamma_{2} \eta\right] \rho
\end{aligned}
$$

We observe that one root of equation (30) never belongs to the interval $[0,1]$, being $r=1+\frac{2 d \gamma_{2}}{N a_{2}}>1$ and so an inner equilibrium is a root of the second degree equation in square brackets in (30). Specific conditions for the existence of an equilibrium with $r \in(0,1)$ can be given. For instance, assuming that $a_{1}>a_{2}$, then the second degree equations has always two real solutions $r_{1}^{*}<r_{2}^{*}$ with $\lim _{\gamma_{2} \rightarrow 0^{+}} r_{1}^{*}=1^{-}$, so that, by continuity, a sufficiently low cost coefficient $\gamma_{2}$ ensures the existence of at least one equilibrium with $r \in(0,1)$.

\section{Stability analysis}

The Jacobian matrix

$$
J\left(X_{1}, X_{2}, r\right)=\left[\begin{array}{ccc}
\rho-\frac{r N a_{1}}{2 \gamma_{1}}-\frac{2 \rho}{K} X_{1}-\frac{\alpha \beta X_{2}}{\left(\alpha+X_{1}\right)^{2}} & -\frac{\beta X_{1}}{\alpha+x_{1}} & -\frac{N a_{1} X_{1}}{2 \gamma_{1}} \\
\frac{\eta \alpha \beta X_{2}}{\left(\alpha+X_{1}\right)^{2}} & \frac{\eta \beta X_{1}}{\alpha+X_{1}}-d-\frac{(1-r) N a_{2}}{2 \gamma_{2}} & \frac{N a_{2}}{2 \gamma_{2}} X_{2} \\
\frac{r(1-r) a_{1}^{2}}{4 \gamma_{1}} & -\frac{r(1-r) a_{2}^{2}}{4 \gamma_{2}} & (1-2 r)\left(\frac{a_{1}^{2}}{4 \gamma_{1}} X_{1}-\frac{a_{2}^{2}}{4 \gamma_{2}} X_{2}\right)
\end{array}\right]
$$

at the global extinction equilibrium $S_{0}^{e}=(0,0, r)$ becomes:

$$
J\left(S_{0}^{e}\right)=\left[\begin{array}{ccc}
\rho-\frac{r N a_{1}}{2 \gamma_{1}} & 0 & 0 \\
0 & -d-\frac{(1-r) N a_{2}}{2 \gamma_{2}} & 0 \\
\frac{r(1-r) a_{1}^{2}}{4 \gamma_{1}} & -\frac{r(1-r) a_{2}^{2}}{4 \gamma_{2}} & 0
\end{array}\right]
$$


which is a triangular matrix with a vanishing eigenvalue along the trapping line of equilibria $(r$ axis), stable along the $X_{1}$ axis provided that, as usual, $2 \gamma_{1} \rho<r N a_{1}$. Instead, at the equilibrium $S_{1}^{e}=(K, 0,0)$ with predator's extinction and no prey harvesting, the Jacobian matrix is triangular again

$$
J\left(S_{1}^{e}\right)=\left[\begin{array}{ccc}
-\rho & -\frac{\beta K}{\alpha+K} & -\frac{N a_{1} K}{2 \gamma_{1}} \\
0 & \frac{\eta \beta K}{\alpha+K}-d-\frac{N a_{2}}{2 \gamma_{2}} & 0 \\
0 & 0 & \frac{a_{1}^{2}}{4 \gamma_{1}} K
\end{array}\right]
$$

but the equilibrium is always unstable due to the third eigenvalue which is always positive (unstable along a direction transverse to $X_{1}$ axis, due to the time evolution of $r$ that has the tendency to increase in a neighborhood of the equilibrium). At the equilibrium $S_{2}^{e}=\left(\frac{K}{2 \rho \gamma_{1}}\left(2 \rho \gamma_{1}-N a_{1}\right), 0,1\right)$ with $2 \rho \gamma_{1}>N a_{1}$, where predator is extinct and all fishermen harvest preys, the Jacobian is triangular again and two eigenvalues are always negative. Therefore $S_{2}^{e}$ is unstable if the natural conditions for predators' survival $\frac{\eta \beta X_{1}^{*}}{\alpha+X_{1}^{*}}>d$ hold true, i.e. $K\left(2 \rho \gamma_{1}-N a_{1}\right)(\eta \beta-d)>2 d \rho \gamma_{1} \alpha$, otherwise it is stable. At the equilibrium $S_{3}^{e}=\left(X_{1}^{e}, X_{2}^{e}, 0\right)$, with coexisting preys and predators and no prey harvesting, the Jacobian matrix reads

$$
J\left(S_{3}^{e}\right)=\left[\begin{array}{ccc}
\rho\left(1-\frac{2 X_{1}^{e}}{K}\right)-\frac{\alpha \beta X_{2}^{e}}{\left(\alpha+X_{1}^{e}\right)^{2}} & -\frac{\beta X_{1}^{e}}{\alpha+X_{1}^{e}} & -N \frac{a_{1} X_{1}^{e}}{2 \gamma_{1}} \\
\frac{\alpha \eta \beta X_{2}^{e}}{\left(\alpha+X_{1}^{e}\right)^{2}} & 0 & N \frac{a_{2} X_{2}^{e}}{2 \gamma_{2}} \\
0 & 0 & \frac{a_{1}^{2}}{4 \gamma_{1}} X_{1}^{e}-\frac{a_{2}^{2}}{4 \gamma_{2}} X_{2}^{e}
\end{array}\right]
$$

from which it is straightforward to observe that $\frac{a_{1}^{2}}{4 \gamma_{1}} X_{1}^{e}-\frac{a_{2}^{2}}{4 \gamma_{2}} X_{2}^{e}$ is an eigenvalue, and the other two eigenvalues are solutions of the equation $\lambda^{2}-J_{11} \lambda-J_{12} J_{21}=0$, where $J_{i j}$ is the entry at row $i$-th and column $j$-th of $J\left(S_{3}^{e}\right)$. So, being $-J_{12} J_{21}>0$, the conditions for the asymptotic stability of $S_{3}^{e}$ become

$$
\begin{array}{r}
\frac{a_{1}^{2}}{4 \gamma_{1}} X_{1}^{e}-\frac{a_{2}^{2}}{4 \gamma_{2}} X_{2}^{e}<0 \\
\rho\left(1-\frac{2 X_{1}^{e}}{K}\right)-\frac{\alpha \beta X_{2}^{e}}{\left(\alpha+X_{1}^{e}\right)^{2}}<0
\end{array}
$$

which can be restated, substituting the equilibrium values as

$$
\beta \in\left(\frac{N a_{2}+2 d \gamma_{2}}{2 \gamma_{2} \eta},+\infty\right) \text { and } K \in\left(0, \frac{\alpha\left(N a_{2}+2 \gamma_{2}(d+\beta \eta)\right)}{2 \gamma_{2}(\beta \eta-d)-N a_{2}}\right)
$$

For $\beta \in\left(\frac{N a_{2}+2 d \gamma_{2}}{2 \gamma_{2} \eta},+\infty\right)$ and $K=\frac{\alpha\left(N a_{2}+2 \gamma_{2}(d+\beta \eta)\right)}{2 \gamma_{2}(\beta \eta-d)-N a_{2}}$, the characteristic equation has one negative root and two complex conjugate roots with zero real part, i.e. the equilibrium can undergo a Hopf bifurcation if nondegeneracy conditions are satisfied. A similar analysis holds for the equilibrium $S_{4}^{e}=\left(\widetilde{X}_{1}^{e}, \widetilde{X}_{2}^{e}, 1\right)$.

Finally, for the equilibria (if any) $S_{5}^{e}=\left(\widehat{X}_{1}^{e}, \widehat{X}_{2}^{e}, \widehat{r}\right)$ where both prey and predators are harvested, 
substituting the equilibria conditions in the Jacobian matrix we get

$$
J\left(S_{5}^{e}\right)=\left[\begin{array}{ccc}
\widehat{X}_{1}^{e}\left[\frac{\alpha \beta \gamma_{2} a_{1}^{2} \widehat{X}_{1}^{e}}{\gamma_{1} a_{2}^{2}\left(\alpha+\widehat{X}_{1}^{e}\right)^{2}}-\frac{\rho}{K}\right. & -\frac{\beta \widehat{X}_{1}^{e}}{\alpha+\widehat{X}_{1}^{e}} & -N \frac{a_{1} \widehat{X}_{1}^{e}}{2 \gamma_{1}} \\
\frac{\alpha \eta \beta \gamma_{2} a_{1}^{2} \widehat{X}_{1}^{e}}{\gamma_{1} a_{2}^{2}\left(\alpha+\widehat{X}_{1}^{e}\right)^{2}} & 0 & N \frac{a_{1}^{2} \widehat{X}_{1}^{e}}{2 \gamma_{1} a_{2}} \\
\widehat{r}(1-\widehat{r}) \frac{a_{1}^{2}}{4 \gamma_{1}} & -\widehat{r}(1-\widehat{r}) \frac{a_{2}^{2}}{4 \gamma_{2}} & 0
\end{array}\right]
$$

By applying the Routh-Hurwitz criterion to $J\left(S_{5}^{e}\right)$, we can deduce that no stable equilibrium with $r \in(0,1)$ exists whenever $\frac{\alpha \beta \gamma_{2} a_{1}^{2} \widehat{X}_{1}^{e}}{\gamma_{1} a_{2}^{2}\left(\alpha+\widehat{X}_{1}^{e}\right)^{2}} \geq \frac{\rho}{K}$, whereas, when the reverse inequality holds, i.e. when the biomass equilibrium level $\widehat{X}_{1}^{e}$ belongs to a given interval, stability of the inner equilibrium can be achieved for specific parameter values. The equilibrium can undergo a Hopf bifurcation for $J_{21}\left(J_{12} J_{11}+J_{13} J_{32}\right)+J_{31}\left(J_{13} J_{11}+J_{12} J_{23}\right)=0 . \square$

\section{References}

[1] G. Hardin, The tragedy of the commons, Science, 162 (1968) 1243-1247.

[2] C.W. Clark, Mathematical Bioeconomics: The optimal management of renewable resources, Second ed., Wiley-Intersciences, New York, 1990.

[3] R. D. Fischer, L. J. Mirman, Strategic dynamic interaction: Fish wars, Journal of Economic Dynamics and Control, 16 (1992) 267-287.

[4] R. D. Fischer, L. J.Mirman, The Compleat Fish Wars: Biological and Dynamic Interactions, Journal of Environmental Economics and Management, 30 (1996) 34-42.

[5] G.I. Bischi, F. Lamantia, Harvesting dynamics with protected and unprotected areas, Journal of Economic Behavior and Organization 62 (2007) 348-370.

[6] G. I. Bischi, F. Lamantia, L. Sbragia, Strategic interaction and imitation dynamics in patch differentiated exploitation of fisheries, Ecological Complexity, 6 (2009) 353-362.

[7] G.I. Bischi, F. Lamantia, L. Sbragia, Competition and cooperation in natural resources exploitation: an evolutionary game approach, In: Carraro, C., Fragnelli, V. (Eds.) Game Practice and the Environment, Edward Elgar Publishing, Northampton, (2004) 187-211.

[8] G. I. Bischi, F. Lamantia, D. Radi, Multi-species exploitation with evolutionary switching of harvesting strategies, (2012) submitted.

[9] F.J. Weissing, Evolutionary stability and dynamic stability in a class of evolutionary normal form games. In R. Selten (Ed.), Game Equilibrium Models I: Evolution and Game Dynamics. Springer, 1991.

[10] M. S. Branicky, Stability of hybrid systems: State of the art, Proc. IEEE Conf. on Decision and Control, San Diego, CA, (1997) 120-125. 
[11] A. Rejniak, R. A. Anderson, Hybrid models of tumor growth, WIREs Syst Biol Med, 3 (2011) $115-125$.

[12] S. Gao, D. Xie, L. Chen, Pulse vaccination strategy in a delayed sir epidemic model with vertical transmission, Discrete and continuous dynamical systems-Series B, 7 (2007) 77-86.

[13] R. Goebel, R.G. Sanfelice, A.R. Teel, Hybrid dynamical systems, IEEE Control Systems Magazine, 29 (2009) 28-93.

[14] A. Xepapadeas, Regulation and Evolution of Compliance in Common Pool Resources. The Scandinavian Journal of Economics, (2005) 107(3), 583-599.

[15] G. F. Gause, La théorie mathématique de la lutte pour la vie, Paris, Hermann, 1935.

[16] Yu. M. Svirezhev, D. O. Logofet, Stability of biological communities, Mir, Moscow, 1983.

[17] D. L. DeAngelis, Dynamics of nutrient cycling and food webs, Chapman \& Hall, New York, 1992.

[18] M. L. Rosenzweig, R. H. MacArthur, Graphical representation and stability conditions of predator-prey interactions, The American Naturalist, 97 (1963) 209-223.

[19] C. S. Holling, The functional responce of predators to prey density and its role in mimicry and population regulation, Memoirs of the Entomological Society of Canada, 45 (1965) 1-60.

[20] J. Vandermeer, Loose coupling of predator-prey cycles: entrainment, chaos, and intermittency in the classic MacArthur consumer-resource equations, The American Naturalist, 141 (1993) 687716 .

[21] A. Colombo, F. Dercole, S. Rinaldi, Remarks on metacommunities synchronization with application to prey-predator systems, The American Naturalist, 171 (2008) 430-442.

[22] L. Yunfei, P. Yongzhen, G. Shujing, L. Changguo, Harvesting of a phytoplankton-zooplankton model, Nonlinear Analysis: Real World Applications, 11 (2010) 3608-3619.

[23] T. K. Kar, U. K. Pahari, K. S. Chaudhuri, Conservation of a prey-predator fishery with predator self limitation based on continuous fishing effort, J. Appl. Math. \& Computing, 19 (2005) 311-326.

[24] M. Spence, Product differentiation and welfare, Amer. Econ. Rev, 66 (1976) 407-414.

[25] J. Häckner, A Note on Price and Quantity Competition in Differentiated Oligopolies, Journal of Economic Theory, 93 (2000) 233-239.

[26] F. Szidarovszki and K. Okuguchi. An oligopoly model of commercial fishing. Seoul Journal of Economics, 11:321-330, (1998).

[27] O. De Feo, S. Rinaldi, Yield and dynamics of tritrophic food chains, The American Naturalist, 150 (1997) 328-345. 
[28] V. A. A. Jansen, Regulation of Predator-Prey Systems through Spatial Interactions: A Possible Solution to the Paradox of Enrichment, Oikos, 74 (1995) 384-390.

[29] V. A. A. Jansen, The Dynamics of Two Diffusively Coupled Predator Prey Population, Theoretical Population Biology, 59 (2001) 119-131.

[30] J. Weibull, Evolutionary Game Theory, Cambridge, MA: The M.I.T. Press, 1995.

[31] J. Hofbauer, K. Sigmund, Evolutionary Games and Population Dynamics, Cambridge University Press, 1998.

[32] J.-P. Aubin, J. Lygeros, M. Quincampoix, S. S. Sastry, and N. Seube, Impulse differential inclusions: a viability approach to hybrid systems, IEEE Transactions on Automatic Control, 47(1) (2002) 2-20.

[33] W. M. Haddad, V. Chellaboina, S. G. Nersesov, Impulsive and Hybrid Dynamical Systems:Stability, Dissipativity, and Control, Princeton University Press, 2006.

[34] M. L. Rosenzweig, The Paradox of Enrichment, Science, 171 (1971) 385-387.

[35] L. Oksanen, S. D. Fretwell, J. Arruda, P. Niemela, Exploitation ecosystems in gradients of primary productivity, The American naturalist, 118 (1981) 240-261.

[36] P. A. Abrams, Effect of increased productivity on the abundances of trophic levels, The American Naturalist 141 (1993) 351-371.

[37] J. Guckenheimer, P. Holmes, Nonlinear Oscillations, Dynamical Systems and Bifurcations of Vector Fields. Springer-Verlag, New York, 1983. 